\title{
Human pose, hand and mesh estimation using deep learning: a survey
}

\author{
Mukhiddin Toshpulatov ${ }^{1}$ (D) Wookey Lee ${ }^{1} \cdot$ Suan Lee $^{2} \cdot$ \\ Arousha Haghighian Roudsari ${ }^{3}$
}

Accepted: 19 October 2021 / Published online: 1 January 2022

(c) The Author(s) 2021

\begin{abstract}
Human pose estimation is one of the issues that have gained many benefits from using state-of-the-art deep learning-based models. Human pose, hand and mesh estimation is a significant problem that has attracted the attention of the computer vision community for the past few decades. A wide variety of solutions have been proposed to tackle the problem. Deep Learning-based approaches have been extensively studied in recent years and used to address several computer vision problems. However, it is sometimes hard to compare these methods due to their intrinsic difference. This paper extensively summarizes the current deep learning-based $2 D$ and $3 D$ human pose, hand and mesh estimation methods with a single or multi-person, single or double-stage methodology-based taxonomy. The authors aim to make every step in the deep learning-based human pose, hand and mesh estimation techniques interpretable by providing readers with a readily understandable explanation. The presented taxonomy has clearly illustrated current research on deep learning-based $2 D$ and $3 D$ human pose, hand and mesh estimation. Moreover, it also provided dataset and evaluation metrics for both $2 D$ and $3 D H P E$ approaches.
\end{abstract}

Keywords $3 D$ pose estimation - Generator - Discriminator - Loss function · Deep neural network $\cdot$ Deep learning $\cdot$ Mesh estimation $\cdot$ Evaluation metric $\cdot$ Dataset

\section{Human pose estimation}

Human pose estimation $(H P E)$ recently has been significantly studied in the $A I$ research community. $H P E$ aims to obtain the posture of the human body from given sensor inputs. $H P E$ is a crucial research study in the modern computer vision field and has been implemented into many applications, such as human-computer interaction $(H C I)[14,20]$, healthcare, motion analysis, virtual reality (VR) and augmented

Wookey Lee

trinity@inha.ac.kr

Extended author information available on the last page of the article 
reality (AR). Novel deep learning (DL) models outperformed classical methods in several research fields such as image classification, semantic segmentation, object and face detection. Subsequently, the HPE has also achieved outstanding achievement utilizing the $D L$ methods. With the rapid advancement in the HPE research field, this work tracks recent progress and summarizes their achievements to provide readers with a clear understanding of current research on $D L$-based $H P E$.

Several review works are published on the $2 D$ and $3 D H P E$ topics. The authors of [20], in their survey study, presented only $2 D H P E$ methods, including singleor multi-person approaches. In [14], the authors provide a review on $2 D$ and $3 D$ HPE methods with their related categories. Zheng et al. [124] also covers a similar context with [124]. However, all the mentioned studies include papers from the early DL implementation to the $H P E$ field. Unlike the existing survey papers, this research work thoroughly summarizes the recently published $D L$-based $2 D H P E$ and $3 D H P E$. One of the key points of the current research that previous ones did not includes $3 D$ human hand, and mesh estimation approaches, which are important for a high rapidly growing $A I$ applications. We have used the novel taxonomy, which differs from the previous ones, and it covers state-of-the-art $3 D$ human hand with mesh estimation approaches. We have also discussed several recently published papers in great detail, which revolutionized in the related research field. This research addresses the weaknesses of the existing survey studies in the HPE field, covering the key points of $H P E$ methods, advantages and drawbacks, extensive analysis of their experimental implementation details. We have reviewed the recent $H P E$ milestone, which the previous survey studies did not cover. In addition, it includes recently published $D L$-based $3 D$ human hand and mesh estimation approaches, which are rapidly growing and gaining a great attraction among the $A I$ researchers.

In this survey study, we have reviewed and discussed the recently published research works in the related field in two main divisions: 2D HPE (Sect. 2) and $3 D$ human pose estimation (Sect. 3). Each of them is also divided into subcategories based on their respective characteristics. Table 1 shows a taxonomy of all the reviewed papers during this research process by their related categories.

Our contribution through this survey study and advantages of the research work from the previous similar surveys are: Recently published novel $D L$-based $2 D H P E$ and $3 D H P E$ methods including $3 D$ human hand and mesh estimation approaches are extensively reviewed; Provided a taxonomy of all reviewed approaches by a category corresponding to $2 D$ single or multiple $H P E$ and $3 D$ single or multiple $H P E$, covering single or double stage, model-based or model-free subcategories. Provided extensive performance evaluation of $2 D H P E$ and $3 D$ human hand, pose, mesh estimation approaches; Described mainly used datasets and widely used evaluation metrics used in $2 D H P E$ and $3 D$ human hand, pose, mesh estimation; Reviewed various types of human pose, hand and mesh estimation applications, such as computer gaming, video surveillance, movies and animation, human and computer interaction, self-driving, $A R / V R$, medical assistance and healthcare; Presented vital points of the state-of-the-art 2D HPE and $3 D$ human pose, hand and mesh estimation approaches extensively compared their pros and cons, input and output data, used dataset, backbone, and loss function experimental implementation details and evaluation measures. Also, it presented an insightful discussion of $2 D H P E$ and $3 D$ human pose, 
Table 1 Sections of deep learning-based human pose estimation approaches [14]

\begin{tabular}{|c|c|c|c|}
\hline Division & Sub-division & Section & Sub-section \\
\hline \multirow[t]{4}{*}{$2 D H P E$} & $2 D$ Single & Regression-based & $\begin{array}{l}\text { - Direct prediction } \\
\text { - Multi-task }\end{array}$ \\
\hline & & Detection-based & - Network design \\
\hline & 2D Multiple & Top-down & $\begin{array}{l}\text { - Coarse-to-fine } \\
\text { - Bounding box refinement }\end{array}$ \\
\hline & & Botttom-up & $\begin{array}{l}\text { - Two-stage } \\
\text { - Single-stage } \\
\text { - Multi-task }\end{array}$ \\
\hline \multirow[t]{4}{*}{$3 D H P E$} & $3 D$ Single & Model-free & $\begin{array}{l}\text { - Single-stage } \\
\text { - Two-stage }\end{array}$ \\
\hline & & Model-based & - SMPL-based \\
\hline & & Depth-based & $\begin{array}{l}\text { - Generative } \\
\text { - Discriminative }\end{array}$ \\
\hline & $3 D$ Multiple & & $\begin{array}{l}\text { - Bottom-up } \\
\text { - Top-down } \\
\text { - SMPL-based }\end{array}$ \\
\hline
\end{tabular}

hand and mesh estimation methods regarding open research issues with key challenges in the $H P E$ field, including future research direction.

The paper is organized as follows. Section 1 Human Pose Estimation describes the briefly introduction to the research field with existing problems and includes Sect. 1.1 Recent advancements, Sect. 1.2 Impactful Past Papers and Sect. 1.3 Traditional Human body models. Section 2 extensively describes $2 D$ HPE approaches, dividing them into related categories and given a detailed description of some stateof-the-art methods. Section 3 covers $3 D$ HPE method including single and multiview approaches. It also describes in detail several novel methods which made the revolution in the research field. Section 4 discusses $3 D$ human hand and mesh estimation approaches in detail, as hand and mesh estimation is important and gaining tremendous interest among the researchers in the $A I$ community. The Datasets and Evaluation metrics used in the related field are given and discussed with great details in Sect. 5. Section 6 presents Open issues and challenges in the HPE research community while Sect. 7 gives Summary and Conclusion of this survey study. And Sect. 8 gives the Future research directions in $H P E$ research field.

\subsection{Recent advancements}

Despite the significant progress and remarkable performance of $H P E$, challenges such as occlusion, lacking training data and the depth ambiguity still cause difficulties that need to be overcome. Moreover, compared with 2D HPE, obtaining precise $3 D$ pose annotations is much more complicated. For $3 D H P E$ from $2 D$ data, the main difficulty is depth ambiguities. Researchers have employed inertial measurement units (IMUs), depth sensors and radiofrequency devices as a solution. 
However, these methods are usually not considered as being cost-effective and need special-purpose hardware [124]. A novel Bayesian formulation of Capsule networks $[88,93]$ was implemented for estimating the $3 D$ human pose from a single $R G B$ image. The obtained result was that the pose is given by the $3 D$ coordinates of 17 joints in a human pose skeleton. $3 D$ pose restoration from $2 D$ input is an ill-posed optimization problem and should be regularized. They chose $\mathrm{J}$ capsules with size $\mathrm{S}$ as 512 and 8, respectively. Each capsule is cloned $K=17$ times to predict 17 human pose joints. Another fascinating recent approach is Deep High-Resolution Representation Learning for $H P E$, where the authors proposed that the network maintains high-resolution representations through the whole process [44]. The proposed model starts from a high-resolution subnetwork as the initial stage. It then gradually adds high-to-low-resolution subnetworks one by one to form more stages and connects the multi-resolution subnetworks in parallel. They repeat these multi-scale unions. Each high-to-low resolution representation receives information from other parallel representations, leading to rich, high-resolution representations. The authors argue that the predicted key point heatmap is potentially more accurate and spatially more precise. Moreover, they have empirically demonstrated the effectiveness of the proposed approach through the superior pose estimation results over the $C O C O$ and the MPII dataset.

Multi-person $H P E$ is an attractive and compared with the single-person $H P E$ is a challenging task. Existing methods are mainly based on two-stage and generally suffer from low efficiency. A single-stage-based model, namely Single-stage multiperson Pose Machine(SPM) [71], was proposed to simplify the pipeline and enhance the efficiency for multi-person human pose estimation. The authors propose a Structured Pose Representation $(S P R)$ to unify human body instance and joint positions. The developed $S P M$ based on $S P R$ directly predicts structured poses for multi-person in a single stage. Moreover, the proposed approach offers a more compact pipeline and an attractive efficiency advantage than previous state-of-the-art ones. Even though the single-stage paradigm aims to simplify the multi-person pose estimation and receives much attention, they still have low performance due to the difficulty of regressing various full-body poses from a single feature vector. Unlike previous solutions involving complex heuristic designs, Shi et al. [97] presented a simple and effective solution by employing instance-aware dynamic networks. Specifically, they propose an instance-aware module to adaptively adjust (part of) the network parameters for each instance. The authors argue that the proposed approach can significantly increase the capacity and adaptive-ability of the network for recognizing various human poses while maintaining a compact end-to-end trainable pipeline. The extensive experiments on the $M S$-COCO dataset significantly improve existing single-stage methods and make a better balance of accuracy and efficiency compared to the state-of-the-art two-stage HPE approaches.

\subsection{Impactful past papers}

$H P E$ is defined as the problem of localization of human joints [122] in images or videos or searching for a specific pose in the space of all articulated poses, such 
as illustrated in Fig. 1. It has already been widely exploited in Action recognition, Animation, Gaming applications, such as a very popular Deep Learning app HomeCourt, which uses Pose Estimation to analyze Basketball player movements. Realistic 3D HPE aims to localize semantic key points [3] of single or multiple human bodies in $3 D$ space. It is an crucial element for human behavior understanding, activity recognition with various applications, such as augmented reality or humancomputer interaction. Even though it has been studied for decades in the computer vision field, it has attracted significant research interest due to the introduction of low-cost depth cameras [55, 75, 117].

Convolutional neural networks $(C N N s)$ [59, 68, 76, 89, 106, 108]-based methods outperform existing ones in HPE from a single depth map and achieved noticeable performance improvement. Even though they achieved significant advancement in $3 D$ HPE , they still suffer from inaccurate analysis because of severe self-occlusions, highly articulated shapes of target objects, and low-quality depth images. To overcome these issues, Moon et al. [67] proposed the voxel-to-voxel prediction network for pose estimation ( $V 2 \mathrm{~V}$-PoseNet). The proposed method takes a voxelized grid as input and estimates the per-voxel likelihood for each key point. By converting the $2 D$ depth image into a $3 D$ voxelized form as input, the network can see objects' actual appearance without perspective distortion. Moreover, estimating each key point's per-voxel likelihood enables the system to learn the desired task more quickly than the highly nonlinear mapping that estimates $3 D$ coordinates directly from the input. $H P E$ is a vital research field and can be applied to various applications such as action/activity detection, action recognition [41, 54, 120], human tracking [19,
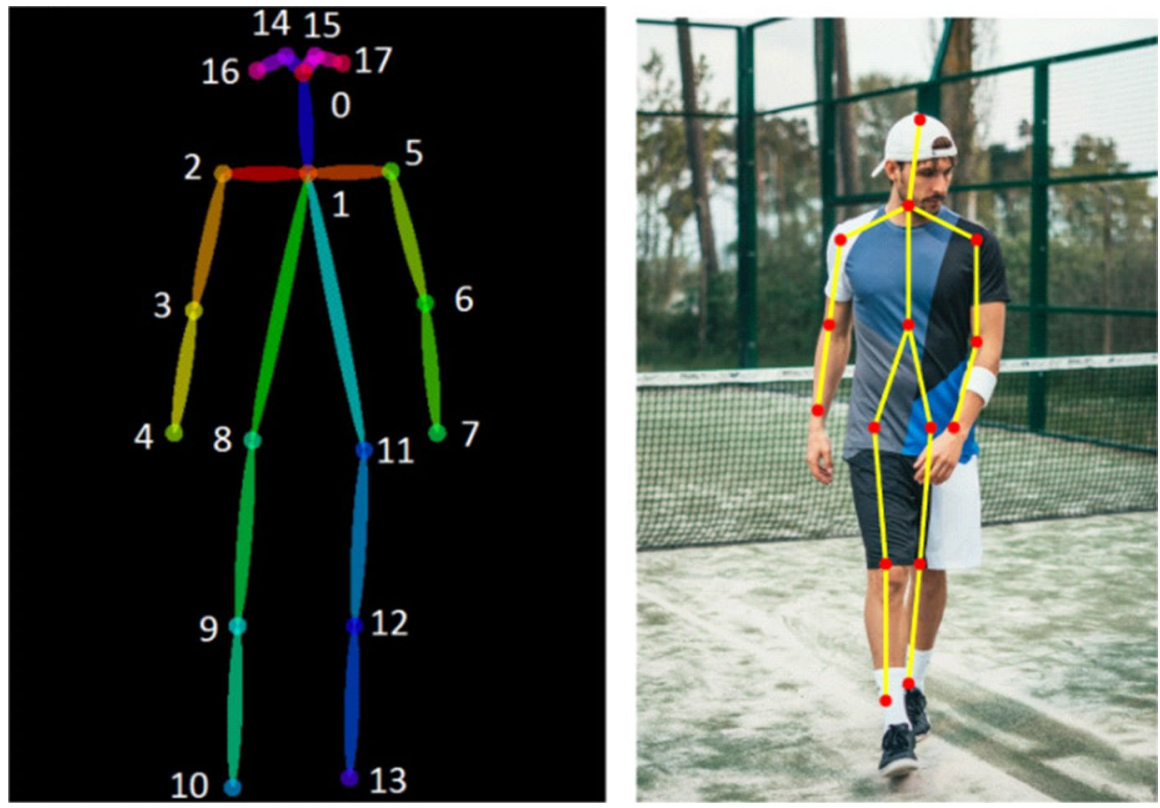

Fig. 1 Pose estimation [3] 
39], virtual reality, video surveillance [2], movies and animation, human-computer interaction [85], self-driving, medical assistance [107] and sports motion analysis. Table 2 shows and describes a list of the applications that can be done with the pose estimations.

\subsection{Human body models}

Modeling of the human body is a key component of HPE. The human body is a flexible and complex non-rigid object. It has many specific characteristics like kinematic structure, surface texture, body shape, the position of body parts or body joints, etc. A mature model for the human body does not necessarily need to contain all human body attributes. When building the human pose model, only the requirements for a specific task need to be met. Based on various application scenarios and levels of representations required, three kinds of human body models are commonly used in HPE. As presented in Fig. 2, these three types include skeleton based, contour based and volume based.

Kinematic model The kinematic model includes a set of joint positions and limb orientations to represent the human body structure. The Pictorial Structure Model $(P S M)$ is a widely used graph model, also known as the tree-structured model. This flexible and intuitive human body model is successfully utilized in $2 D$ HPE and $3 D$

Table 2 A list of the applications that can be done with the pose estimations [14]

\begin{tabular}{|c|c|}
\hline Application & Description \\
\hline Virtual reality & $\begin{array}{l}\text { Promising technology that can be applied in both education and entertain- } \\
\text { ment. Estimation of human posture can further clarify the relationship } \\
\text { between the social and virtual reality world and enhance the interactive } \\
\text { experience }\end{array}$ \\
\hline Video surveillance & $\begin{array}{l}\text { One of the early applications to adopt } H P E \text { technology in tracking, action } \\
\text { recognition, re-identification of people within a specific range }\end{array}$ \\
\hline Movies and animation [42] & $\begin{array}{l}\text { Generation of various vivid digital characters is inseparable from the } \\
\text { capture of human movements. A cheap and accurate social motion } \\
\text { capture system can better promote the digital entertainment develop- } \\
\text { ment industry }\end{array}$ \\
\hline Human-computer interaction & $\begin{array}{l}H P E \text { is very important for computers and robots better to understand } \\
\text { people's identification, location, and action. With humans' posture, } \\
\text { computers and robots can efficiently execute instructions and be more } \\
\text { intelligent }\end{array}$ \\
\hline Self-driving & $\begin{array}{l}\text { Advanced self-driving cars with } H P E \text { can respond more appropriately } \\
\text { to pedestrians and offer more comprehensive interaction with traffic } \\
\text { coordinators }\end{array}$ \\
\hline Medical assistance & $\begin{array}{l}H P E \text { can provide physicians with quantitative human motion information, } \\
\text { especially for rehabilitation training and physical therapy }\end{array}$ \\
\hline Sports motion analysis & $\begin{array}{l}\text { Estimating players' posture in sports videos can further obtain the sta- } \\
\text { tistics of athletes' indicators. } H P E \text { can provide a quantitative analysis } \\
\text { of action details. Instructors can make more objective evaluations of } \\
\text { students with } H P E\end{array}$ \\
\hline
\end{tabular}




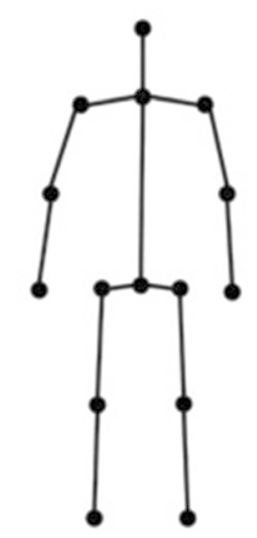

skeleton-based model

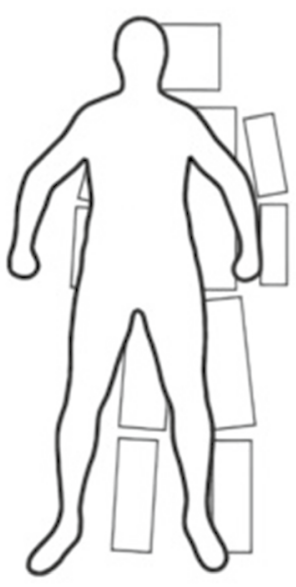

contour-based model

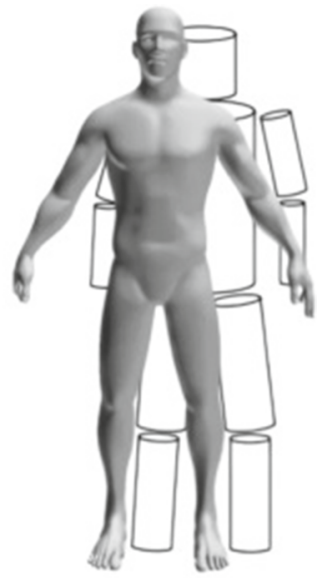

volume-based model

Fig. 2 Three types of models for human body modeling [124]

HPE. Moreover, the kinematic model has the advantage of flexible graph representation even though it is limited in representing texture and shape information.

Planar Model Besides the kinematic model, to capture the relations between different body parts, the planar model represents the shape and appearance of a human body. In the planar model, body parts are usually represented by rectangles approximating the human body contours.

Volumetric Model With the increasing interest in 3D HPE, many human body models have been proposed for a wide variety of human body shapes. It is commonly used in deep learning-based $3 D H P E$ methods for recovering $3 D$ human mesh.

SMPL The skinned Multi-Person Linear model (SMPL) is a skinned vertex-based model, which depicts a wide range of human body shapes. It can be modeled with natural pose-dependent deformations representing soft-tissue dynamics. There are 1786 high-resolution $3 D$ scans of various poses, including template mesh in SMPL. These $3 D$ scans are used to learn how people deform with various poses by optimizing the blend weights, the mean template shape, pose-dependent blend shapes, and the regressor from vertices to joint locations. The existing rendering engines are compatible with $S M P L$ and easy to utilize; therefore, it is broadly adopted in $3 D$ HPE methods.

DYNA Dynamic Human Shape in Motion (DYNA) model represents realistic softtissue motions for different human body shapes. A low-dimensional linear subspace approximates motion-related soft-tissue deformation. For predicting the low-dimensional linear coefficients of soft-tissue motion, the whole body's velocity and acceleration, the body part's angular velocities and accelerations, and the coefficients of the soft-tissue shape are used. DYNA utilizes the body mass index (BMI) to generate deformations for different shaped people. 


\section{2D human pose estimation approaches}

Some solution methods of the $2 D H P E$ task have been reviewed in this section. $2 D$ Human pose estimation has received significant attention recently due to its various applications in the real world. It aims to locate the human body parts from images or videos automatically. All the human parts in the given input image or video should be detected, and the key points of the same person, even in a crowded scene, should be associated. DL-based 2D HPE methods are capable of extracting more sufficient features from metadata. Such methods have resulted in excellent performances and outperformed the non-deep state-of-the-art methods with a considerable margin. Although the deployment of $D L$ in the $H P E$ field is relatively new, several notable works on this issue have been proposed. This section aims to present a comprehensive overview of state-of-the-art $D L$-based $2 D$ human pose estimation methodologies.

\section{1 $2 D$ single-person pose estimation}

$2 D$ Single-person HPE refers to the task of localizing human skeletal key points of a person from the given input image or video frame. There have been proposed several traditional research approaches where was exploited handcrafted feature extraction methods-developing the novel $D L$ technique has been widely implementing into the 2D HPE research field also. DeepPose [105] was the first significant paper that applied Deep Learning to $2 D H P E$. It achieved SOTA performance and exceeded the existing models. DeepPose formulates the pose estimation as a CNN-based regression task toward body joints. Also, the pose estimates are refined by using a cascade of regressors to obtain a better result. The proposed approach does pose reasoning in a holistic manner. That is, even if certain joints are hidden, they can be estimated. The authors argue that $C N N s$ naturally provide this sort of holistic reasoning and demonstrate strong results. The key point of the proposed model is implementing the refinement of the predictions using cascaded regressors. The initial coarse pose is refined, and a better estimate is achieved. Images are cropped around the predicted joint and fed to the next stage. In this way, the subsequent pose regressors see higher resolution images and thus learn finer scales, which ultimately leads to higher precision. In [105], the Cartesian coordinates of body joints are directly estimated using a multi-stage deep network and produced state-of-the-art achievement. Multi-stage $C N N$ also progressively enlarges receptive fields and refines the pose estimation result. In addition, it is trainable with a graphical model. The $C N N$ estimated $2 D$ heatmaps [63] for each joint, and they were exploited as the unary term for the model. In [70], a stacked hourglass network was proposed, which repeats downsampling and upsampling to exploit multi-scale information effectively. Chu et al. [18] attempted to enhance the stacked hourglass network [70] by integrating it with a multi-context attention mechanism. In [13], an iterative error feedback-based HPE system was proposed. Ke et al. [43] presented a multi-scale structure-aware network to achieve 
a leading position in the publicly available $H P E$ benchmark. Mask $R$ - $C N N$ [17] was proposed to perform human detection and key point localization in a single model. The proposed model crops human features from a feature map via the differentiable RoIAlign layer. The schematic view of the proposed DeepPose system is represented in Fig. 3. It consists of an AlexNet backed (8 layers) with an extra final layer, which outputs $2 n$ coordinates- $-\left(x_{i}, y_{i}\right) * 2$, where $i \in\{1,2, \ldots, n\}$, and $n$ is total number of joints. For training, the model uses $L_{2}$ loss for regression and applies refinement of the predictions using cascaded regressors, resulting in achieving better estimates. Images are cropped around the predicted joint and fed to the next stage; in this way, the subsequent pose regressors see higher resolution images and thus learn features for finer scales which ultimately leads to higher precision.

\subsubsection{Regression-based methods}

There have been proposed several research works related to regression model predict human joint coordinates from the input image or video frame. AlexNet is one of the initial networks for deep learning-based 2D HPE approaches due to their simplistic architecture and remarkable performance. It was first trained to learn joint coordinates from full input images in a very straightforward manner. A cascade structure of multi-stage refining regressors was employed to refine the previous stage's cropped images and showed improved performance. It was also applied for predicting the human pose in the videos using a sequence of concatenated frames as an input. Networks handling multiple closely related tasks of the human body may learn various features to improve the prediction of joint coordinates of the human pose. The AlexNet multi-task framework was also employed to handle the joint coordinate prediction task from the given input images regression. We briefly describe the layers of the AlexNet network as it plays a significant role in our research domain.

Figure 4 shows that the Alexnet has eight layers with learnable parameters. It consists of five layers with a combination of max-pooling layers followed by three fully connected layers and use a Relu activation in each of these layers except the output one. It was found that using the Relu as an activation function speeds up the training by almost six times. It also uses the dropout layers that prevent overfitting. The model is trained on the ImageNet dataset, with 14 million images.
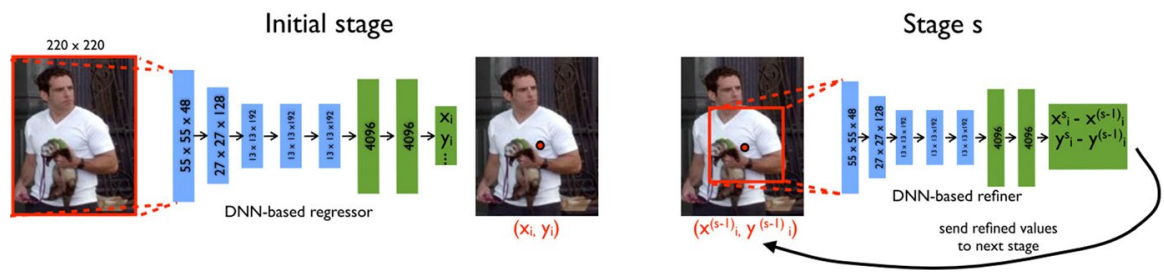

Fig. 3 Schematic view of the proposed DeepPose system [105] 


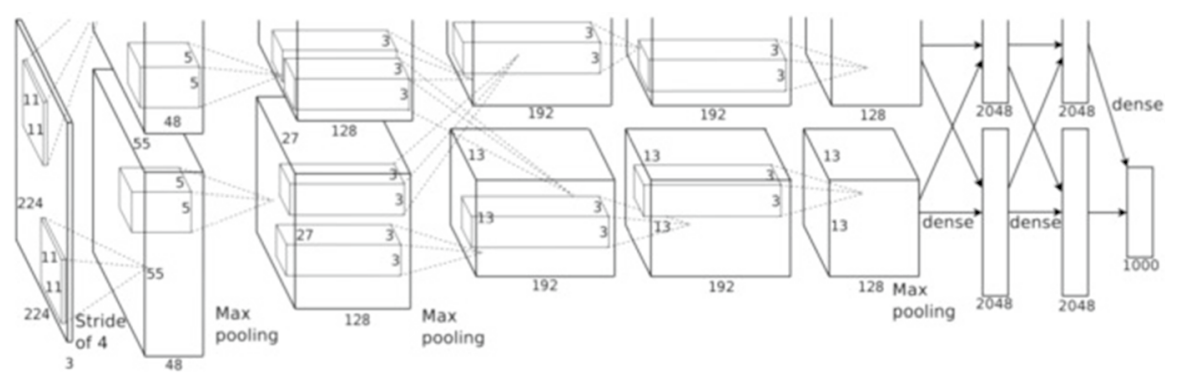

Fig. 4 AlexNet system [20]

\subsection{D multi-person pose estimation}

The multi-person pose estimation has two main approaches: Top-down approach and Bottom-up approach (Fig. 5).

Top-down approach The top-down approach relies on a human detector that predicts bounding boxes of humans. The detected human image is cropped and fed to the pose estimation system. In other words, first is a person detection stage followed by the key point detection stage. These approaches still dominate the leaderboard of public benchmark datasets like MS COCO10 dataset $[52,78]$ and can be summarized based on the following aspects:

- Context modeling

- Effective training strategy

- Post-processing techniques

$[15,34,80,102,112,115]$ researches are based on the top-down approach. Chen et al. [15] introduced a cascaded pyramid network whose cascaded structure refines an initially estimated pose by focusing on hard key points. Xiao et al. [115] proposed a simple pose estimation network that consists of a deep backbone network and several upsampling layers. This model achieved state-of-the-art performance based on simple network architecture on the commonly used benchmark. Papandreou et al. [81] proposed $2 D$ offset vectors and $2 D$ heatmaps for each joint. They fused the estimated vectors and heatmaps to generate highly localized heatmaps.

Bottom-up approach The bottom-up approach localizes all human body key points in an input image and assembles them using proposed clustering algorithms in each work. In other words, this approach directly detects all key points from the picture and associates them with similar person occurrences. Bottom-up approaches are usually faster than top-down methods. [12, 36, 48, 69, 70] works are based on the bottom-up approach.

A novel method called DeepCut [84] formulated the assignment of the detected key points to each person in a given input image as an integer linear program. It improves the performance by introducing image-conditioned pair-wise terms. Part affinity fields $(P A F s)$ [12] exposed the association between human body key points 


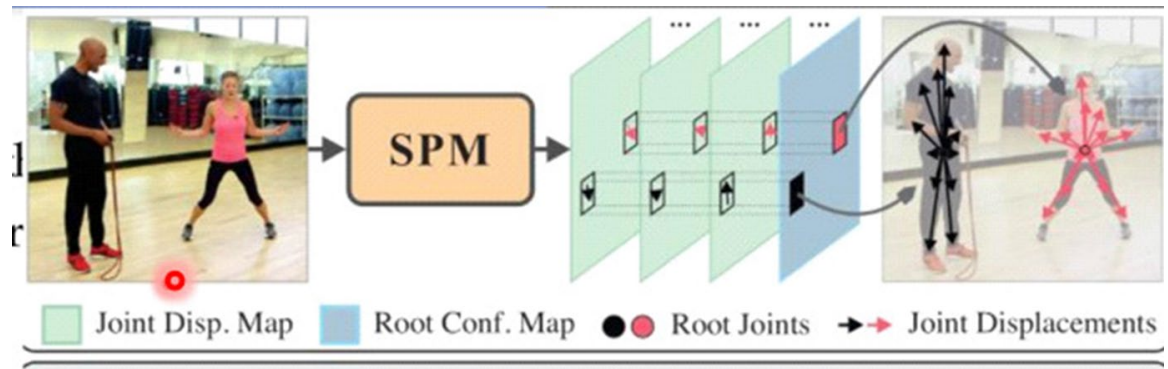

(b) Existing Two-Stage Solution

Top-Down Strategy

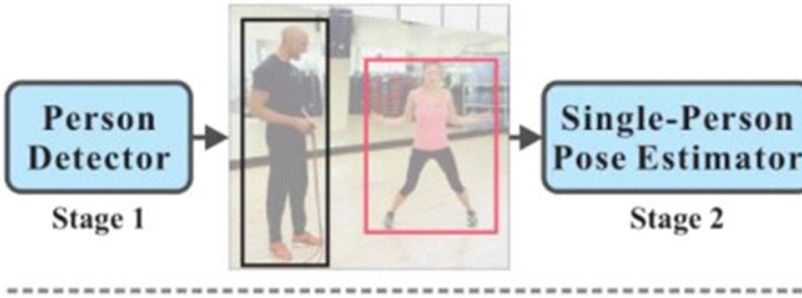

Bottom-Up Strategy

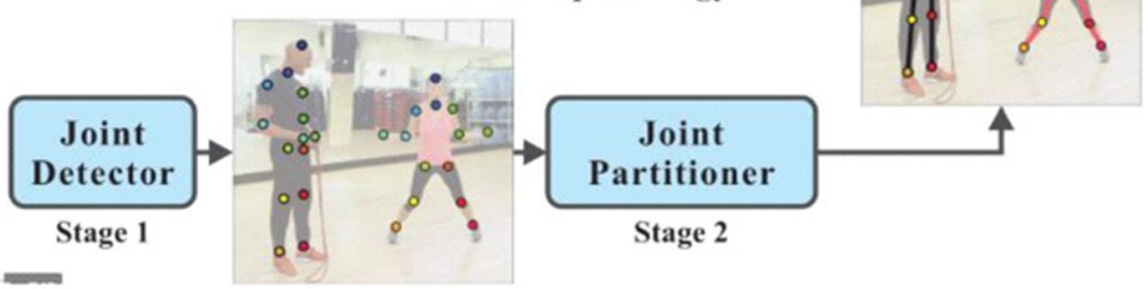

Fig. 5 Two-staged approaches of multi-person pose estimation [71]

directly. Authors assembled the localized key points of all the people in the input image by using the estimated PAFs. Newell et al. [69] proposed a pixel-wise tag value to assign localized key points to a certain human. Pose residual network (PRN) [48] is a pose estimation model that can assign detected key points to each person while having the ability to jointly perform key point detection, person detection and person segmentation. Chen et al. [15] proposed a cascaded pyramid network $(C P N)$ which consists of two structures: GlobalNet; RefineNet.

GlobalNet GlobalNet is based on a deep backbone network and upsampling layers with skip connections (Fig. 5).

RefineNet RefineNet is built to refine the estimation results from the GlobalNet by focusing on hard key points. 


\section{$2.32 D$ human pose refinement}

Many methods endeavored to refine the approximated key point for more realistic representation. Chen et al. [15], Newell et al. [70], Bulat and Tzimiropoulos [9-11] exploited an end-to-end trainable multi-stage architecture-based network. The utilized model at each stage tries to refine the pose estimation results of the previous stage via end-to-end learning. The model proposed in [13] iteratively estimated error feedback from a shared weight model. The previous iteration's output error feedback is transformed into the input pose of the next iteration, which is repeated several times for progressive pose refinement. These methods combine pose estimation and refinement into a single model. The refinement module is dependent on the estimation, and models have a refinement module with a different structure. Hence, they are not guaranteed to work appropriately, combining with other estimation methods. Moon et al. [65] proposed a pose refinement method independent of the estimation, where the results can be consistently improved regardless of the prior pose estimation method. Fieraru et al. [25] proposed a post-processing network to refine the pose estimation results of other methods, which is conceptually similar to [65]. The proposed model synthesizes pose for training and uses simple network structure that estimates refined heatmaps and offset vectors for each joint. It follows ad hoc rules $[8,96]$ to generate input pose, while the previous [65] approach is based on actual error statistics obtained through empirical analysis.

In [65], a refinement network PoseFix was proposed to estimate a refined pose from a tuple of an input image and a human pose. It takes pose estimation results of any other method with an input image and outputs an elegant pose. Multi-stage architectures have mainly performed pose refinement. However, this approach is positively related to the pose estimation model and requires careful refinement design. The authors proposed a model-agnostic pose refinement method that does not depend on the pose estimation model. The proposed model takes the input pose in a coarse form and estimates the refined pose in a finer form. The coarse input pose enables the model to focus not only on an exact location of the input pose but also around it. Besides, the finer form of the output pose enables to localize the location of the pose. PoseFix can be applied to the pose estimation results of any single- or multi-person pose estimation method. Figure 6 shows a pose refinement pipeline of the PoseFix.

The PoseFix model refines the input $2 D$ coordinates of all the persons' human body key points in an input image. It is built based on the top-down pipeline, which processes a cropped human image's tuple and a given pose estimation result of that person. In the training stage, the input pose is synthesized on the ground-truth pose realistically and diversely. In the testing stage, the pose estimation results of any other methods can be the input pose to the system. The overall pipeline of the PoseFix is described in Fig. 7. 

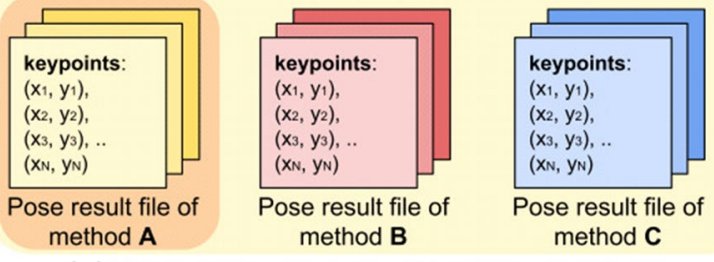

(a) Pose estimation results of any methods
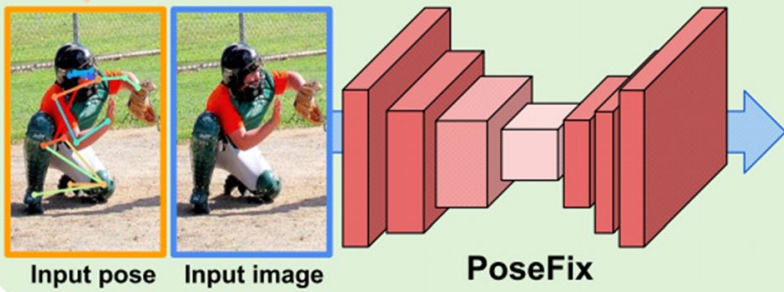

PoseFix

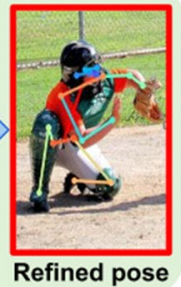

(b) Pose error fix procedure of the PoseFix

Fig. 6 Testing pipeline of the PoseFix [65]

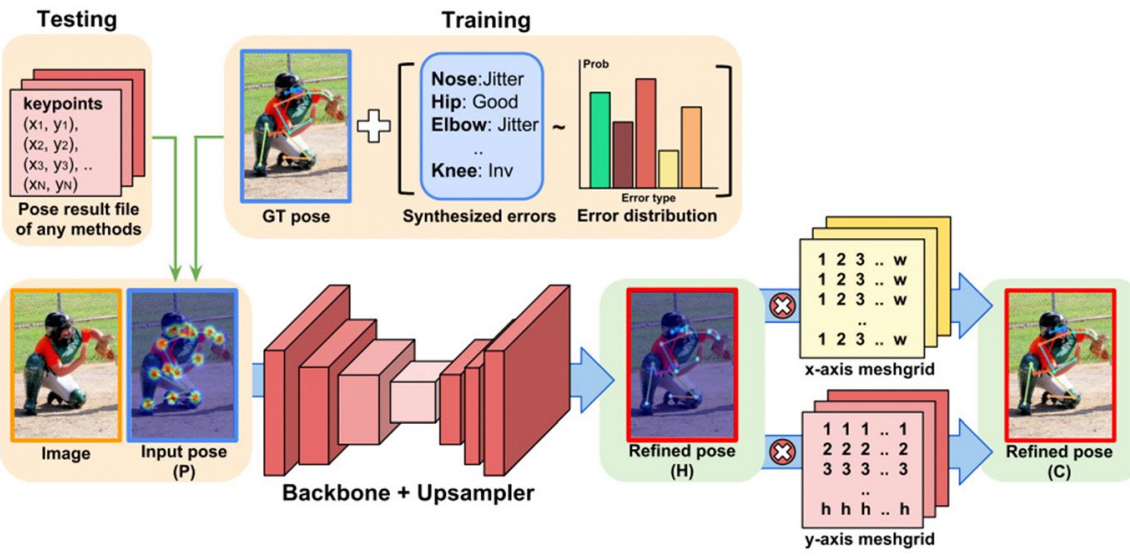

Fig. 7 Overall pipeline of the PoseFix [65]

\section{3D human pose estimation approaches}

$3 D H P E$ predicts locations of the human body joints in $3 D$ space from the given input image sources. The progress of $3 D H P E$ from the input data can further improve the multi-view $3 D H P E$ in constrained environments. This section will focus on the $D L$-based methods that estimate $3 D$ human pose from the $2 D R G B$ images and videos, including $3 D$ single-person and $3 D$ multi-person pose estimation approaches. Most of the previous 3D HPE methods are designed for a single-person 
case. They crop the human area in an input image with a ground-truth bounding box that is predicted from a human detection model. The cropped patch of a human body is fed into the $3 D$ pose estimation module, which then estimates each key point's $3 D$ location. Estimating the absolute camera-centered coordinate of each key point is difficult because the models take the cropped image as input. Many approaches estimate the relative $3 D$ pose to reference point in the body to manage this problem. The final $3 D$ pose is obtained by adding the $3 D$ coordinates of the root to the estimated root-relative $3 D$ pose. Prior information on the bone length [83] or the ground-truth [102] has been commonly used to localize the root.

\subsection{D single-person pose estimation}

$3 D H P E$ is challenging as it needs to predict the depth information of human body joints. Preparation of the training data is also not easy. The existing datasets for $3 D$ $H P E$ are obtained under constrained environments. In $3 D$ single person pose estimation cases, the bounding box of the person is usually provided. $3 D$ single person pose estimation is divided mainly into two: model-free and model-based categories.

Model-free methods The model-free $3 D$ single-person pose estimation methods do not employ human body models as the predicted target. According to the input type, Current $3 D$ single-person pose estimation methods can be categorized into three approaches: single-stage approach; two-stage approach; depth-based approach.

Single-stage approach The single-stage approach takes an $R G B$ image as an input for the $3 D$ human pose estimation model and directly localizes the $3 D$ body key points from the input data. The compositional loss was proposed [100] to consider the joint connection structure. Soft-argmax operation was exploited [102] to obtain the $3 D$ coordinates of body joints in a differentiable manner. Moreover, they introduced a multi-task framework that jointly trains both the pose regression and body part detectors. Tekin et al. [104] modeled high-dimensional joint dependencies by adopting an auto-encoder structure. Pavlakos et al. [83] extended the $U$-net shaped network to estimate a $3 D$ heatmap for each joint. They used a coarse-to-fine approach to boost the performance. Martinez et al. [58] proposed a simple network that consists of consecutive fully-connected layers, which lifts the $2 D$ human pose to the $3 D$ space. Sharma et al. [95] combined a generative model and depth ordering of joints to predict the most reliable $3 D$ pose corresponding to the estimated $2 D$ pose. The $2 D$ pose-based approach lifts the $2 D$ human pose to the $3 D$ space. Zhao et al. [123] generated a semantic GraphCNN to use spatial relationships between joint coordinates. Choi et al. [16] follow the $2 D$ pose-based approach to make the Pose2 Mesh more robust to the domain difference between the training set's controlled environment and in-the-wild environment of the testing set.

Two-stage approach The two-stage methods utilize the high accuracy of $2 D H P E$. They localize body key points in a $2 D$ space and lift them to a $3 D$ area. Motivated by the recent success of $2 D H P E, 2 D$ pose-based $3 D H P E$ estimation approaches that infer $3 D$ human pose from the intermediately estimated $2 D$ human pose have become a popular $3 D$ HPE solution. Benefiting from the excellent performance of state-of-the-art $2 D$ pose detectors, $2 D$ pose-based $3 D H P E$ approaches generally 
outperform direct image-based $3 D H P E$ approaches. In the beginning stage, off-theshelf $2 D H P E$ models are applied to estimate $2 D$ pose from the input data, and then in the next stage, $2 D$ pose-based $3 D H P E$ model is used to obtain the $3 D$ pose. Martinez et al. [58] proposed a simple network that directly regresses the $3 D$ coordinates of body joints from $2 D$ coordinates. Yang et al. [118] utilized adversarial loss to handle the wild's $3 D$ HPE. Park et al. [82] estimated the initial $2 D$ pose and utilized it to regress the $3 D$ pose. Zhou et al. [125] introduced a geometric loss to facilitate weakly supervised learning of the depth regression module.

Depth-based 3D human pose estimation With the recent success of networks in the image generation process has been demonstrated the use of generative networks to guide the $3 D H P E$ during the training process. Depth-based $3 D H P E$ exploits depth maps from estimated skeletons of the human body. Depth-based $3 D H P E$ methods also rely on: generative models and discriminative models.

Generative models The generative models estimate the posture by finding the similarities between the pre-defined body and input $3 D$ point clouds. The ICP [64] algorithm is usually used for $3 D$ body tracking problems. Template fitting with Gaussian mixture models also was proposed.

Discriminative models The discriminative models directly estimate the positions of body joints without requiring body templates. Conventional discriminative methods are mostly based on random forests. Haque et al. [30] proposed the viewpointinvariant pose estimation method using $C N N$ and multiple recurrent neural network rounds. The proposed approach learns viewpoint-invariant features, which makes the model robust to viewpoint variations.

\subsection{D multi-person pose estimation}

$3 D$ multi-person $H P E$ for crowded scenes is essential in many computer vision applications such as autonomous driving, surveillance, and robotics. However, estimating the $3 D$ human pose from a crowded real-world setting is still challenging. A three-step process is commonly used in the multi-person 3D HPE problem: (1) detecting human body key points; (2) matching people across different views; (3) reconstructing $3 D$ human pose. Unfortunately, the critical second step of matching people across different views is non-trivial. Pose estimation in group pictures with severe occlusions attracts much attention. The $3 D$ multi-person pose estimation from multiview images aims to estimate each key point's $3 D$ coordinate rather than the $2 D$ coordinate on the group image. Some joints may be more relevant to specific actions than others. Attention mechanism has been used to discover informative joints.

Estimation of a human pose can be very useful in many real-world AIoT scenarios, such as rehabilitation exercises monitoring and assessment, dangerous behavior monitoring and human-machine interaction. Some researches have been done on $3 D$ multi-person pose estimation from a single $R G B$ image. Mehta et al. [62] presented a bottom-up approach system. They proposed an occlusion-robust pose-map formulation that supports pose inference for more than one person through PAFs. In [91] 


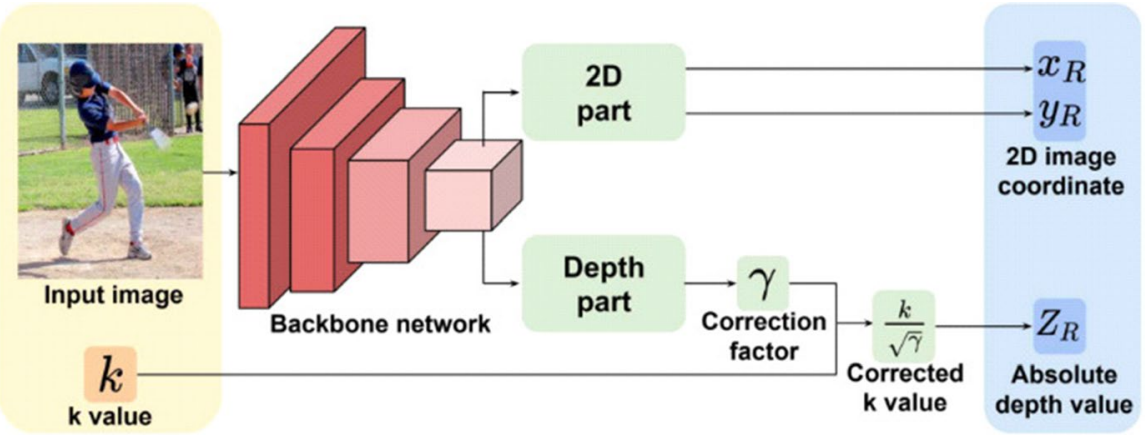

Fig. 8 Network architecture of the RootNet [64]

was introduced a top-down approach called $L C R$-Net. The proposed system consists of: localization part; classification part; regression parts.

Localization part The first localization part detects a human from an input image.

Classification part And the next classification part classifies the detected human into several anchor-poses. The anchor-pose is defined as a pair of $2 D$ and root-relative $3 D$ pose. It is generated by clustering poses in the training set.

Regression part Then, the last regression part refines the anchor-poses.

A novel and general framework was proposed by Moon et al. [64] for 3D multiperson pose estimation from a single $R G B$ image. The presented framework consists of three Networks: human detection DetectNet, $3 D$ human root localization RootNet and root-relative $3 D$ single-person pose estimation PoseNet models. The authors declared that existing human detection and $3 D$ single-person pose estimation models could be plugged into their proposed framework as it is very flexible and easy to use.

DetectNet. Mask R-CNN was exploited as the framework of Detect-Net. Mask $R-C N N$ consists of three parts: backbone; region proposal network; classification head network.

- Backbone. It extracts useful local and global features from the input image by using a deep residual network (ResNet) [23, 31] and feature pyramid network [22, 24, 98].

- Region proposal network. It proposes human bounding box candidates based on the extracted features.

- Classification head network. The RoIAlign layer extracts each proposal's features and passes them to the third part, which is the classification head network. The head network determines whether the given proposal is a human or not and estimates the bounding box refinement offsets.

RootNet. The RootNet localizes the human's root $R=\left(x_{R}, y_{R}, Z_{R}\right)$ from a cropped human image, where $x_{R}$ and $y_{R}$ are pixel coordinates, $Z_{R}$ is absolute depth value. RootNet estimates the $2 D$ image coordinates $\left(x_{R}, y_{R}\right)$ and the human root's depth value separately. The $2 D$ image coordinates are back-projected to the camera-centered coordinate space using the estimated depth value. The image provides sufficient information on 
where the human root is located in the image space. The $2 D$ estimation part can learn to localize it easily. By contrast, estimating the depth only from a cropped human image is difficult because the input does not provide information on the camera and human's relative position. The network architecture of the RootNet is visualized in Fig. 8.

RootNet is trained by minimizing the $L_{1}$ distance between the estimated and groundtruth coordinates. The loss function $L_{\text {root }}$ is defined as follows:

$$
L_{\text {root }}=\left\|R-R^{*}\right\|_{1}
$$

where $*$ indicates the ground-truth.

PoseNet. The PoseNet estimates the root-relative $3 D$ pose from a cropped human image as follows:

$$
P_{j}^{r e l}=\left(x_{j}, y_{j}, Z_{j}^{r e l}\right)
$$

where $j$ is the number of human joints. It was exploited by Sun et al. [102], as a current state-of-the-art method, and it consists of two parts: Backbone; Pose estimation.

- Backbone. The first part, which is the backbone, extracts a useful global feature from the cropped human image using ResNet.

- Pose estimation. It takes a feature map from the backbone part and upsamples it using three consecutive deconvolutional layers with batch normalization layers [5, 37, 74] and $\operatorname{ReL} U$ [35, 53] activation function. A 1-by-1 convolution is applied to the upsampled feature map to produce the $3 D$ heatmaps for each joint. The soft-argmax operation is used to extract the $2 D$ image coordinates $\left(x_{j}, y_{j}\right)$ and the root-relative depth values $Z_{j}^{\text {rel }}$

PoseNet is trained by minimizing the $L_{1}$ distance between the estimated and groundtruth coordinates. The loss function $L_{\text {pose }}$ is defined as follows:

$$
L_{\text {pose }}=\frac{1}{J} \sum_{j=1}^{J}\left\|P_{j}^{r e l}-P_{j}^{r e l *}\right\|
$$

where $*$ indicates the ground truth, and $J$ is the total number of coordinates.
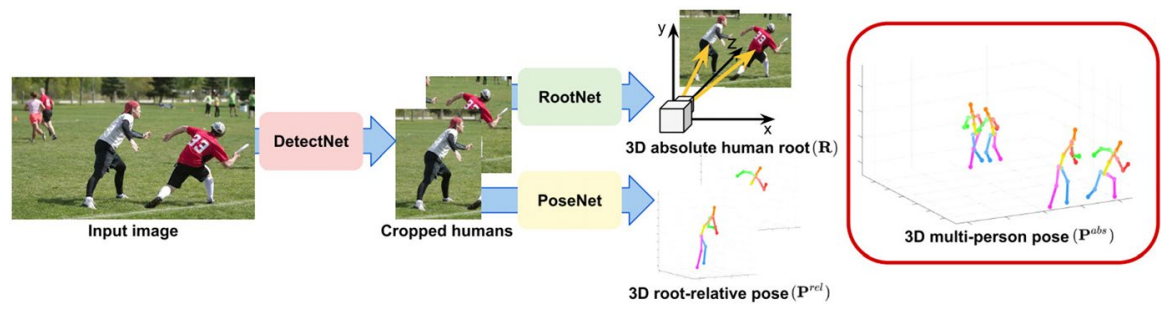

Fig. 9 Overall pipeline of the proposed framework for $3 D$ multi-person pose estimation from a single RGB image [64] 
The network architecture of the proposed work, which consists of three components, is visualized in Fig. 9:

- A human detection network (DetectNet) detects the bounding boxes of humans in an input image.

- The proposed 3D human root localization network (RootNet) estimates the detected human roots' camera-centered coordinates.

- A root-relative $3 D$ single-person pose estimation network (PostNet) estimates the root-relative $3 D$ pose for each detected human.

\subsection{Volumetric representation using depth information}

Wu et al. proposed a depth image's volumetric representation that surpassed the existing hand-crafted descriptor-based methods in $3 D$ shape classification and retrieval problems. Each voxel was represented as a binary random variable and employed a convolutional deep belief network to learn the probability distribution for each voxel. Recent work [116] also represented 3D input data as a volumetric form for $3 D$ object classification and detection. In [67], several types of volumetric representations were proposed to fully utilize the rich source of $3 D$ information and efficiently deal with large amounts of point cloud data. Their presented CNN architecture and occupancy grids outperform state-of-the-arts in several available datasets.

\section{3D human hand and mesh estimation methods}

$3 D$ human pose and mesh estimation models were proposed to recover $3 D$ human joint and mesh vertex locations simultaneously. This is a challenging task due to the depth and scale ambiguity, complexity of the human body and hand articulation. Recent deep learning-based methods have shown distinct performance improvement in solving this problem and outperformed all previous approaches. The deep learning-based methods rely on human mesh models and can be generally categorized into two approaches: model-based approach and model-free approach.

Model-based approach In the model-based approach, a network is trained to predict the model parameters and to generate a human mesh by decoding them $[4,6,7$, 38, 49, 77, 79].

Model-free approach In contrast, the model-free approach regresses a $3 D$ human mesh coordinates directly $[28,50,51]$. Both given approaches compute the $3 D$ human pose by multiplying the output mesh with a joint regression matrix defined in the human mesh models [16, 29, 32, 46, 86, 87, 92, 94].

Deep learning-based $3 D$ human pose and mesh estimation models regress the pose and shape parameters of human mesh models. Even though they recently have shown significant improvement, they still have weaknesses: suffering from domain gap and unapproprite parameters. 
Suffering from domain gap When tested on in-the-wild data, the models suffer from the gap that exists between the controlled and in-the-wild environment data. The data obtained from the controlled environments [7, 33, 60, 90, 113, 114] are considered as valuable train data in $3 D$ human pose and estimation because it contains accurate $3 D$ annotations. However, due to the significant difference in image appearance between the two domains, such as backgrounds and clothes, an imagebased approach cannot fully benefit from the data.

Unapproprite parameters The pose parameters of the human mesh models might not be an appropriate regression target $[50,51]$. The SMPL $[6,56]$ pose parameters represent $3 D$ rotations in an axis-angle, resulting from the non-unique problem (i.e., periodicity). Although scientists $[38,77]$ tried to avoid the periodicity by utilizing a rotation matrix as the prediction target, it still has a non-minimal representation problem. Choi et al. [16] proposed Pose2Mesh as a solution to the above problems. Pose $2 M e s h$ is a graph convolutional network that recovers $3 D$ human pose and mesh from the $2 D$ human pose, in a model-free fashion. It has two advantages over existing methods: $2 D$ poses from controlled and in-the-wild environments, and avoiding the representation issues. Tables 3, 4, 5 give a complete understanding of the discussed models and describe the taxonomy of the models, exploited networks, and experimental details, respectively.

$2 D$ poses from controlled and in-the-wild environments The proposed method benefits from a relatively homogeneous geometric property of the input $2 D$ pose from controlled and in-the-wild environments. They alleviate the appearance of domain gap problem and provide essential geometric information on human articulation. $2 D$ poses can be estimated accurately from in-the-wild images since many well-performing methods $[15,65,99,115]$ are trained on large-scale in-the-wild $2 D$ human pose datasets [1].

Avoiding the representation issues The next advantage is that the proposed method avoids the pose parameters' representation issues while exploiting the human mesh topology. Pose $2 M e s h$ directly regresses the $3 D$ coordinates of mesh vertices using a graph convolutional neural network (Graph $C N N$ ) with graphs constructed from the mesh topology.

Pose2Mesh. Pose2Mesh is designed in a cascaded architecture, which consists of PoseNet and MeshNet. The PoseNet lifts the $2 D$ human pose to the $3 D$ human pose. Moreover, the MeshNet takes both $2 D$ and $3 D$ human poses to estimate the $3 D$ human mesh in a coarse-to-fine manner. The mesh features are initially processed in a coarse resolution and gradually upsampled to a fine resolution during the forward propagation. The overall pipeline of the proposed Pos $2 M e s h$ system is represented in Fig. 10.

\subsection{Depth-based 3D hand pose estimation}

Depth-based $3 D$ hand pose estimation methods can be divided into: generative methods; discriminative methods; hybrid methods.

Generative methods appropriate a pre-defined hand shape and fit it to the input depth image by minimizing hand-crafted cost functions. Particle swam optimization 


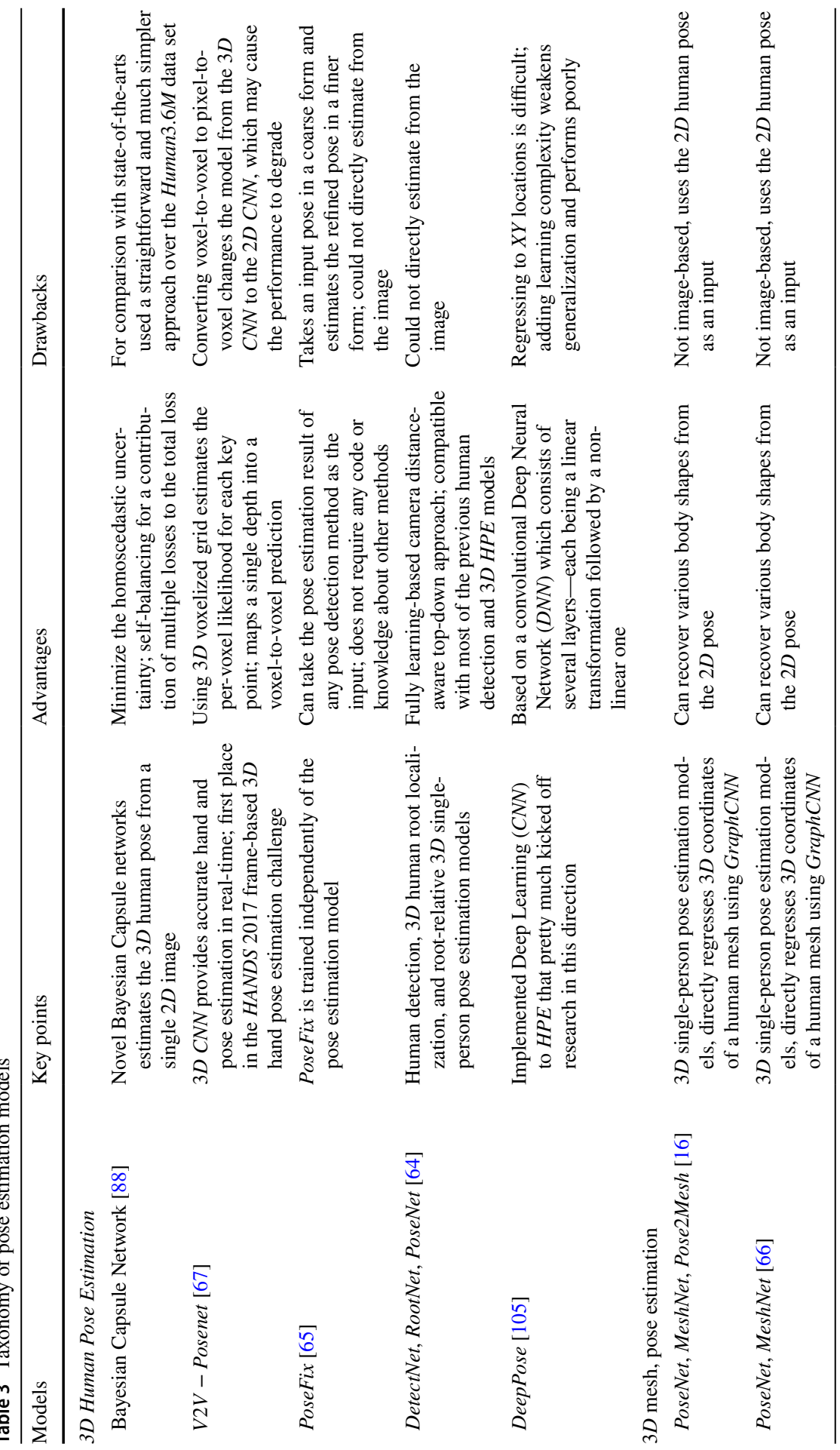




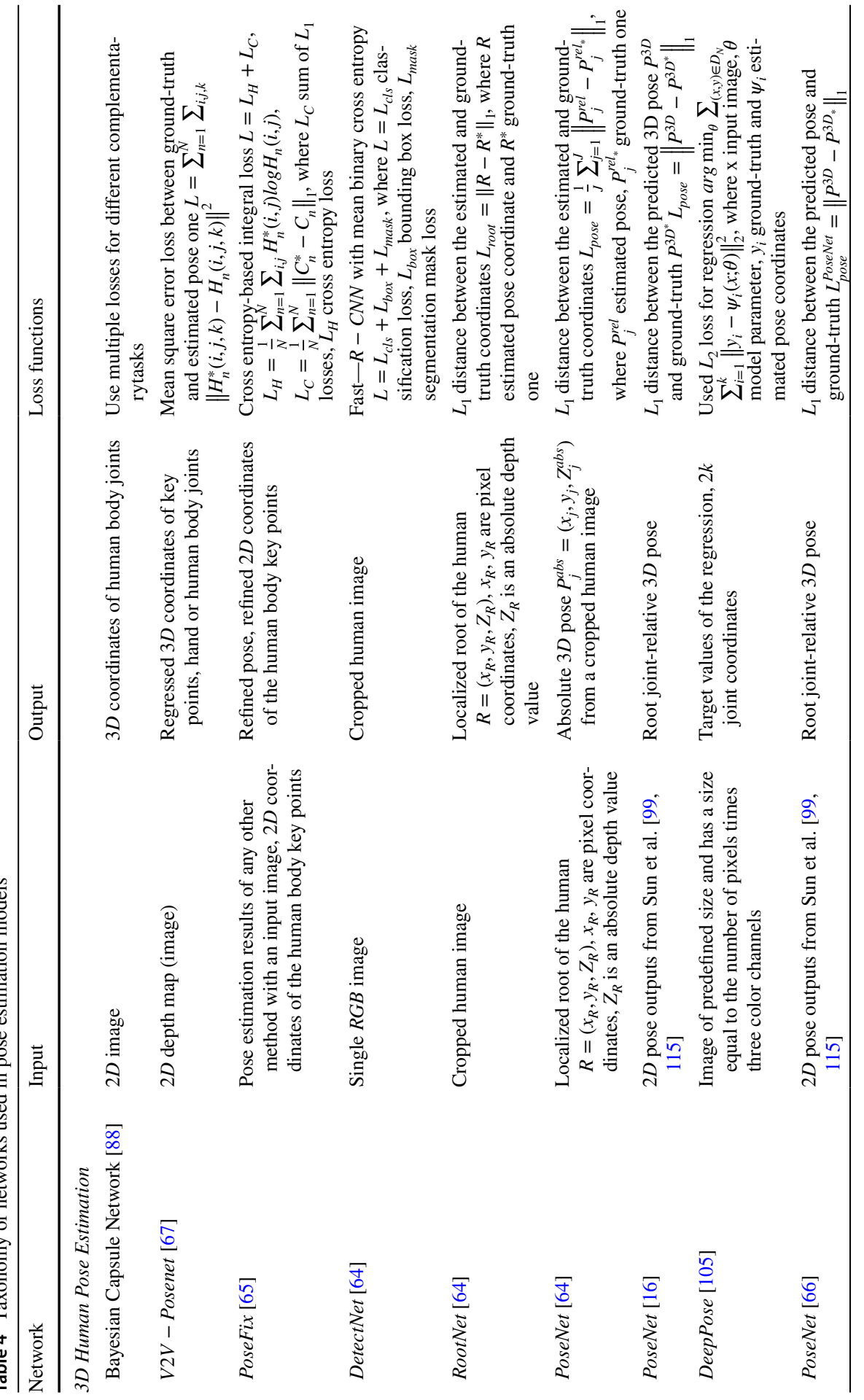




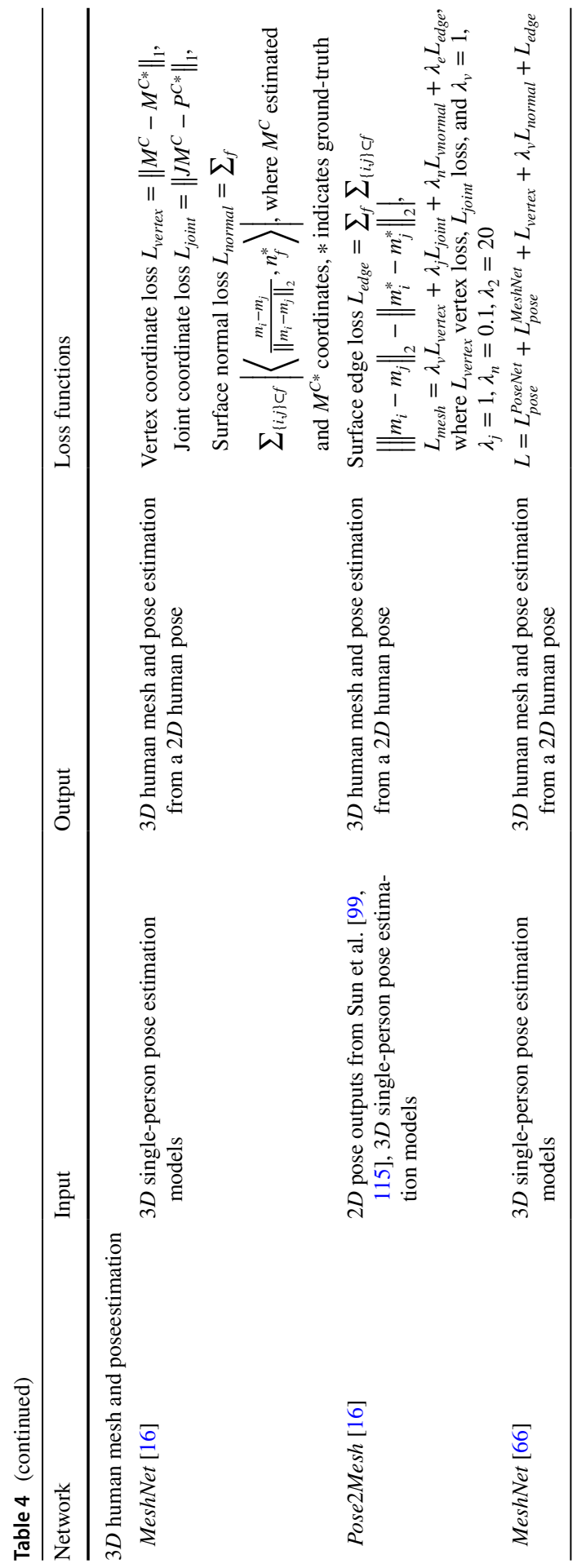




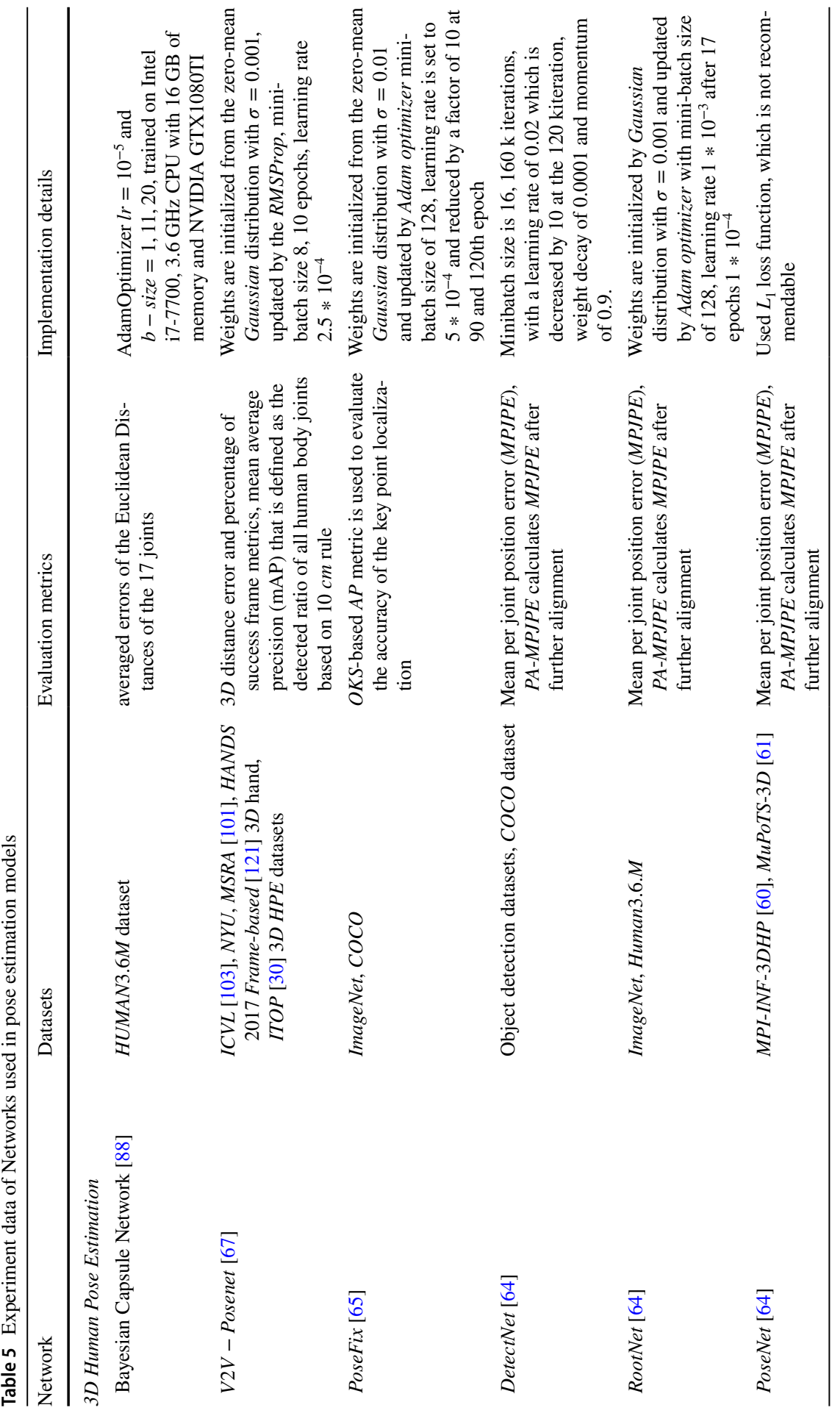




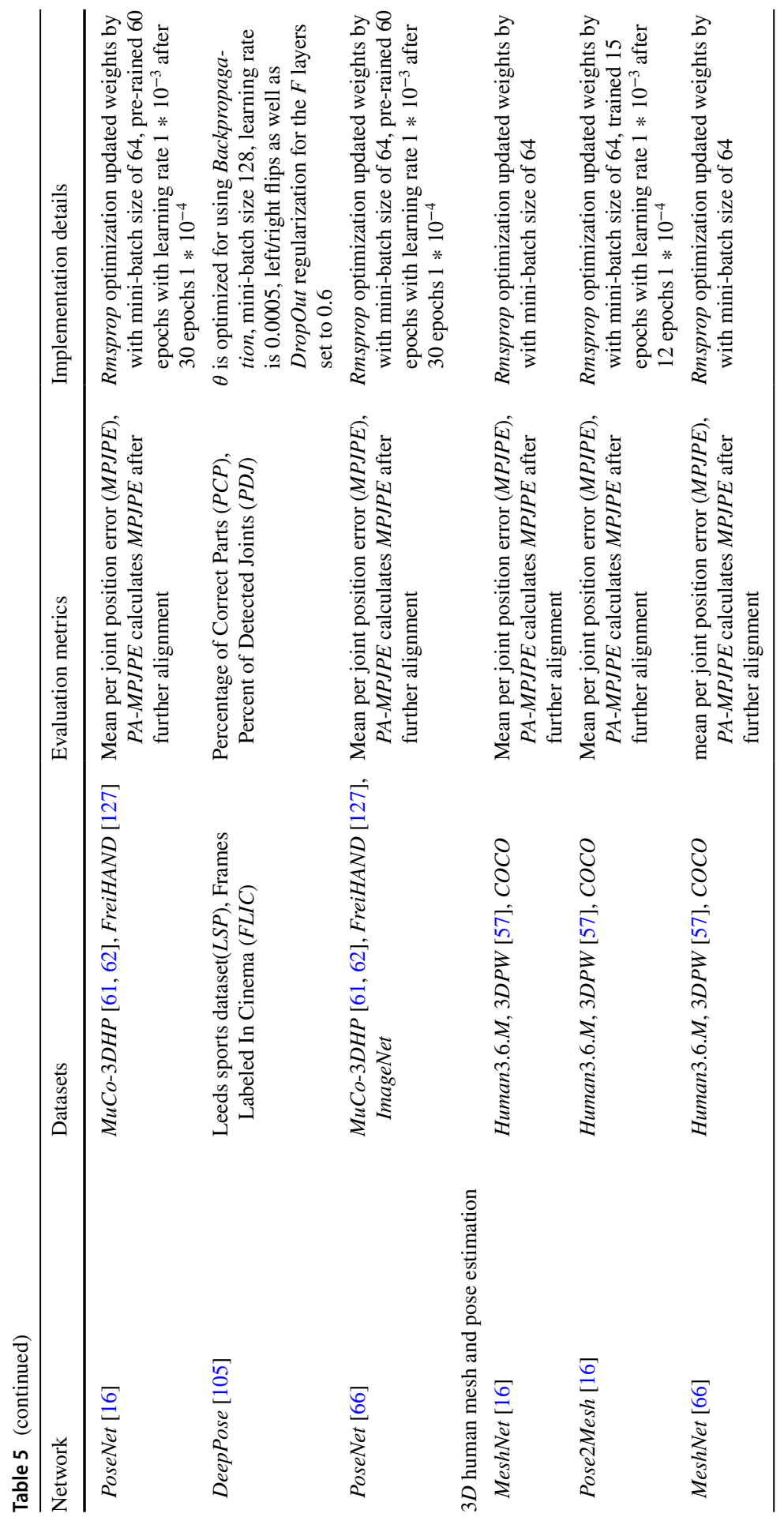




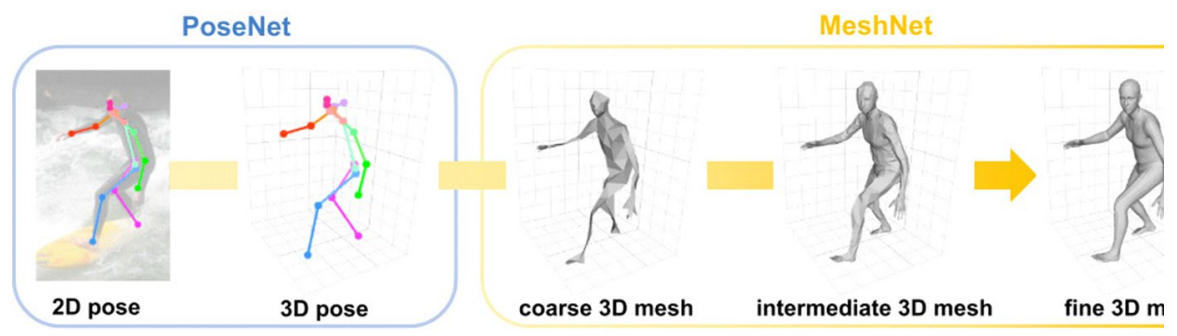

Fig. 10 Overall architecture of the Pose2Mesh network [16]

(PSO), iterative closest point $(I C P)$, and their combination are the familiar algorithms used to obtain optimal hand pose [47] results.

Discriminative methods directly localize hand joints from an input depth map. Random forest-based methods [45] provide quick and precise representation. The $C N N$-based approaches outperform the existing methods and can learn useful features by themselves. $C N N$ primarily was utilized to localize hand key points by estimating $2 D$ heatmaps for each hand joint and then was extended by exploiting multi-view $C N N$ to estimate $2 D$ heatmaps. In [26, 27], the $2 D$ input depth map was transformed to the $3 D$ form and the $3 D$ coordinates were calculated directly via $3 D$ CNN.

Hybrid methods are a combination of the generative and discriminative approach. Oberweger et al. [73] suggested training the discriminative and generative CNNs by a feedback loop. Zhou et al. [126] proposed defining a hand model and estimating the model's parameter then regress to $3 D$ coordinates. Furthermore, in [119], the spatial attention mechanism and hierarchical PSO were utilized. Wan et al. [110] used two deep generative models with a shared latent space and training discriminator to estimate the posterior of the latent pose.

\subsection{D human hand and mesh estimation}

A model-based approach trains a neural network to estimate the human mesh model parameters $[56,92]$. The neural network has been widely used for the $3 D$ human mesh estimation since it does not necessarily require $3 D$ annotation for mesh supervision. Kanazawa et al. [38] used the adversarial loss to regress plausible SMPL parameters. Baek et al. [4] trained $C N N$ to estimate the $M A N O$ model parameters using a neural renderer [40]. Omran et al. [77] introduced training a network with $2 D$ joint coordinates, which takes human part segmentation as input. The advancement of fitting frameworks $[6,83]$ has motivated a model-free approach that estimates human mesh coordinates directly. Researchers could obtain $3 D$ mesh annotation, which is essential for the model-free methods, from in-the-wild data. Ge et al. [26] utilized a GraphCNN to estimate vertices of hand mesh. Kolotouros et al. [50, 51] proposed a GraphCNN, which learns the template body mesh's deformation to the target body mesh. Moon et al. [66] introduced a new heatmap representation, called lixel, to recover $3 D$ human meshes. Choi et al. [16] presented a novel method 


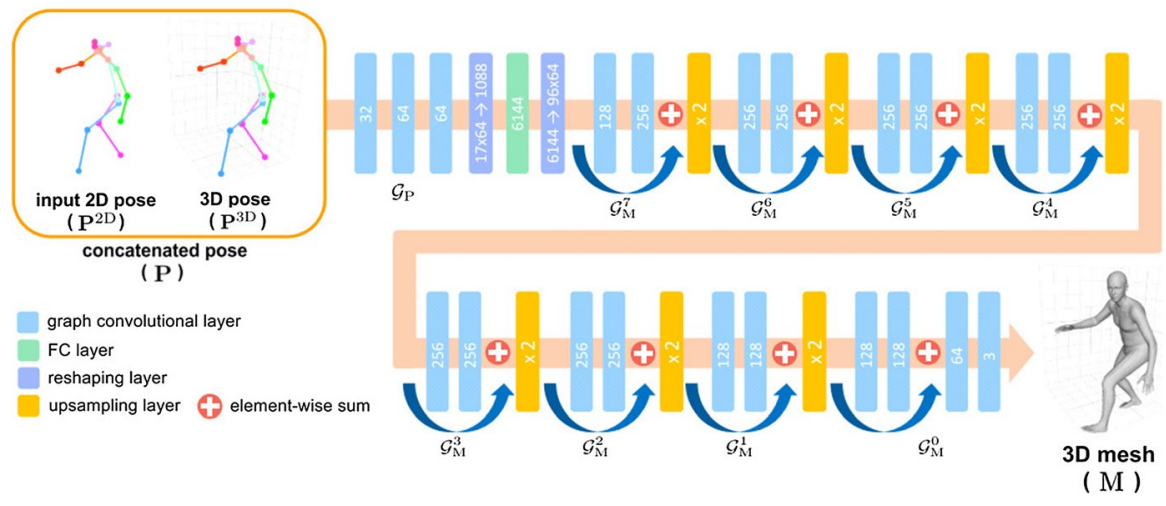

Fig. 11 Network architecture of MeshNet [16]

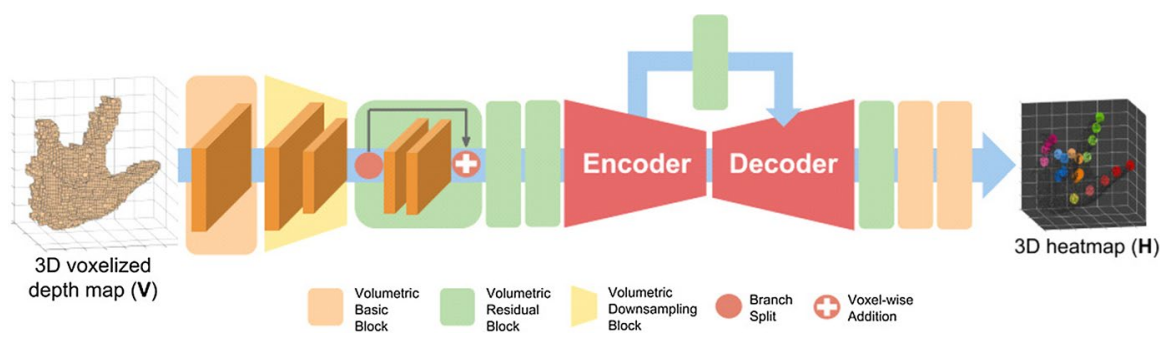

Fig. 12 Overall architecture of the V2V-PoseNet [67]

Pose2Mesh that differs from the previous models, which are image based, in that it uses the $2 D$ human pose as an input. The proposed Pose $2 M e s h$ system can benefit from the data with $3 D$ annotations captured from controlled environments. As described in Fig. 10, it consists of two networks:

- PoseNet

- Meshnet

The network architecture of the MeshNet network model is described in Fig. 11.

\subsection{Input and output representation in $3 D$ hand pose estimation}

The most massive existing $3 D$ hand pose estimation methods from a single depth map are based on taking a $2 D$ depth image and directly regressing $3 D$ coordinates. A $2 D$ depth image was recently converted to a truncated signed distance function-based $3 D$ volumetric form and directly regressed $3 D$ coordinates [21]. In $3 D H P E$ from an $R G B$ image, the per-voxel likelihood for each body key point via $2 D C N N$ was estimated by Pavlakos et al. [83]. The discretized depth value was treated as a channel of the feature map, which resulted in different kernels for each depth value. Moon et al. [67] 
Table 6 Average $3 D$ distance error (mm) [67]

Table 7 Effect of localization refinement and epoch ensemble [67]

\begin{tabular}{lll}
\hline InputOutput & $3 D$ Coordinates & Per-voxel likelihood \\
\hline $2 D$ depth map & $18.85(21.1 \mathrm{M})$ & $13.01(4.6 \mathrm{M})$ \\
$3 D$ voxelized grid & $16.78(457.5 \mathrm{M})$ & $\mathbf{1 0 . 3 7}(\mathbf{3 . 4 M})$ \\
\hline
\end{tabular}

\begin{tabular}{ll}
\hline Methods & $\begin{array}{l}\text { Average } \\
3 D \text { distance } \\
\text { error }\end{array}$ \\
\hline Baseline & $11.14 \mathrm{~mm}$ \\
+Localization refinement & $9.22 \mathrm{~mm}$ \\
+Epoch ensemble & $8.42 \mathrm{~mm}$ \\
\hline
\end{tabular}

proposed to estimate each key point's per-voxel likelihood from the voxelized input. They exploited the $3 D$ fully convolutional network, and it was declared as the first model to generate voxelized output from voxelized input using $3 D C N N$ for $3 D$ hand pose estimation. To estimate the $3 D$ coordinates of all key points, $2 D$ depth images were converted to $3 D$ volumetric forms. Then, $V 2 V-P o s e N e t$ takes the $3 D$ voxelized data and estimates each key point's per-voxel likelihood (Fig. 12).

\subsection{Refining target object localization}

To localize key points, such as hand or human body joints, a cubic box that contains the hand or human body in $3 D$ space is essential. This cubic box is usually placed around the reference point obtained using ground-truth joint position or the center of mass after simple depth thresholding around the hand region. However, in the real-world applications, using the ground-truth joint part is impractical. Moreover, the usage of center of mass calculated by simple depth thresholding does not guarantee that the object is correctly contained in the acquired cubic box due to the error in the center-of-mass calculations in cluttered scenes. When different items are near the target one, the simple depth thresholding method cannot filter the other objects correctly. So, the computed center-of-mass cubic box becomes incorrect. These weaknesses were overcome by training a simple $2 D C N N$; then, $[72,73]$ obtained a valid reference point. The network takes a depth image, whose reference point is calculated by the simple depth thresholding, and outputs $3 D$ offset. The refined reference point can be obtained by adding the network's output offset value to the calculated one. Table 6 shows converting the input image type from the $2 D$ depth map to $3 D$ voxelized form (from $2 D C N N$ to $3 D C N N$ ) substantially improves performance.

The power of the localization-refining procedure and the epoch ensemble are described in Table 7. 


\subsection{GraphCNN for mesh processing}

In current methods, a mesh is considered as a graph structure. It is processed using the GraphCNN as it can fully exploit mesh topology. GraphCNN is utilized to learn a deformation from an initial ellipsoid mesh to the target object mesh in a coarse-tofine manner [111]. Verma et al. [109] introduced a graph convolution operator and evaluated it on the correspondence problem. GraphCNN-based VAE was also proposed, which learns a latent space of the human face meshes hierarchically.

\section{Datasets and evaluation metrics used in HPE}

Datasets and evaluation protocols play a significant role in $D L$-based HPE. They are essential for fair comparison of different algorithms and bring more challenges and complexity through their expansion and improvement. With the maturity of commercial motion capture systems and crowdsourcing services, recent datasets are no longer limited by the data quantity or lab environments. This section will discuss some publicly available datasets for $2 D$ and $3 D \mathrm{HPE}$, with the most used evaluation metrics.

\subsection{Datasets for 2D human pose estimation}

Early created datasets for $2 D H P E$ contain images with relatively simple backgrounds. However, $D L$-based models are unsuitable for these datasets because the number of images is too small for training. The common datasets used in $D L$-based approaches include MSCOCO, MPII, LSP, FLIC, Pose Track, and AI Challenger, which contain more images in more complicated scenes. The HPE datasets, such as FLIC and LSP, are relatively small and only contain specific activity categories. The images in the FLIC dataset are collected from Hollywood movies. The LSP dataset images are from sports scenes. Other datasets, such as AIChallenger and MSCOCO, are bigger in both size and number of image categories.

\subsection{Datasets for 3D human pose estimation}

In contrast with $2 D H P E$ datasets, acquiring accurate $3 D$ annotation for $3 D H P E$ datasets is challenging. It requires motion capture systems such as MoCap and wearable IMUs. Therefore, many $3 D H P E$ datasets are created in constrained conditions.

HumanEva dataset contains seven calibrated video sequences with ground-truth $3 D$ annotation captured by a commercial MoCap system. It consists of four subjects performing six everyday actions: walking, jogging, gesturing, throwing and catching a ball, boxing, and combo. Human3.6M is the mainly used dataset for $3 D H P E$ from monocular images and videos. It consists of 11 professional actors performing 17 activities such as smoking, taking photos, talking on the phone and etc. The dataset contains 3.6 million $3 D$ human poses with $3 D$ ground-truth annotation captured by an accurate marker-based MoCap system. TNT15 dataset consists of synchronized 
data streams from eight $R G B$ cameras and ten $I M U$ s. It has been recorded in an office environment. It records four actors performing five activities: walking, running on the spot, rotating arms, jumping and skiing, and dynamic punching. The dataset contains about 13k frames, including binary segmented images obtained by background subtraction, $3 D$ laser scans and registered meshes of each actor. MPI$I N F-3 D H P$ dataset was collected with a marker- less multi-camera MoCap system and includes both indoor and outdoor scenes. It contains over 1.3 M frames from 14 different views. Eight subjects are recorded performing eight activities such walking/standing, exercise, sitting, crouch/reach, on the floor, sports, miscellaneous. TotalCapture dataset was captured indoors with eight calibrated $H D$ video cameras. There are four male and one female subjects performing four diverse performances, repeated three times. The variation and body motions within the acting and freestyle sequences are very challenging with actions such as yoga, giving directions, bending over and crawling performed in both the train and test data.

Furthermore, the MARCOnI dataset contains sequences in a variety of uncontrolled indoor and outdoor scenarios. They vary according to different data modalities captured, in the numbers and identities of actors to track, the complexity of the motions, the number of cameras used, the existence and number of moving objects in the background and the lighting conditions. Cameras differ in the types, hence the frame resolutions, and the frame rates. Panoptic dataset was captured with a markerless motion capturing using multiple view systems. It contains 65 sequences of social interaction with 1.5 million $3 D$ skeletons. The provided annotations include $3 D$ key points, cloud points, optical flow, etc. $3 D P W$ dataset was captured using a single hand-held camera in natural environments. $3 D$ annotations are estimated from $I M U$ s. All subjects in the dataset are provided with $3 D$ scans. It consists of 60 video sequences with periodic actions, including walking in the city, going upstairs, having coffee, taking the bus, etc. The datasets were collected with MoCap systems. Table 8 shows popular $3 D H P E$ datasets which we have described above.

\subsection{Evaluation metrics used in 2D HPE}

Different datasets have different features and task requirements (single/multi-pose). Therefore, several metrics are used evaluate the performance in $2 D H P E$, which is tricky due to many factors that need to be considered. We will describe some of the commonly used metrics in the following.

Percentage of Correctly estimated body Parts $(P C P)$ metric evaluates stick predictions, and it was used in early research studies. $P C P$ reports the localization accuracy for human limbs. A human limb is correctly localized if its two endpoints are within a threshold from the corresponding ground truth endpoints. Besides, a mean $P C P$, some limbs $P C P$, such as the torso, upper legs, lower legs, upper arms, forearms, head, are also reported. Moreover, percentage curves for each limb can be obtained with the threshold variation in the metric. The similar metrics $P C P m$ use $50 \%$ of the mean ground-truth segment length over the entire test set as a matching threshold. 


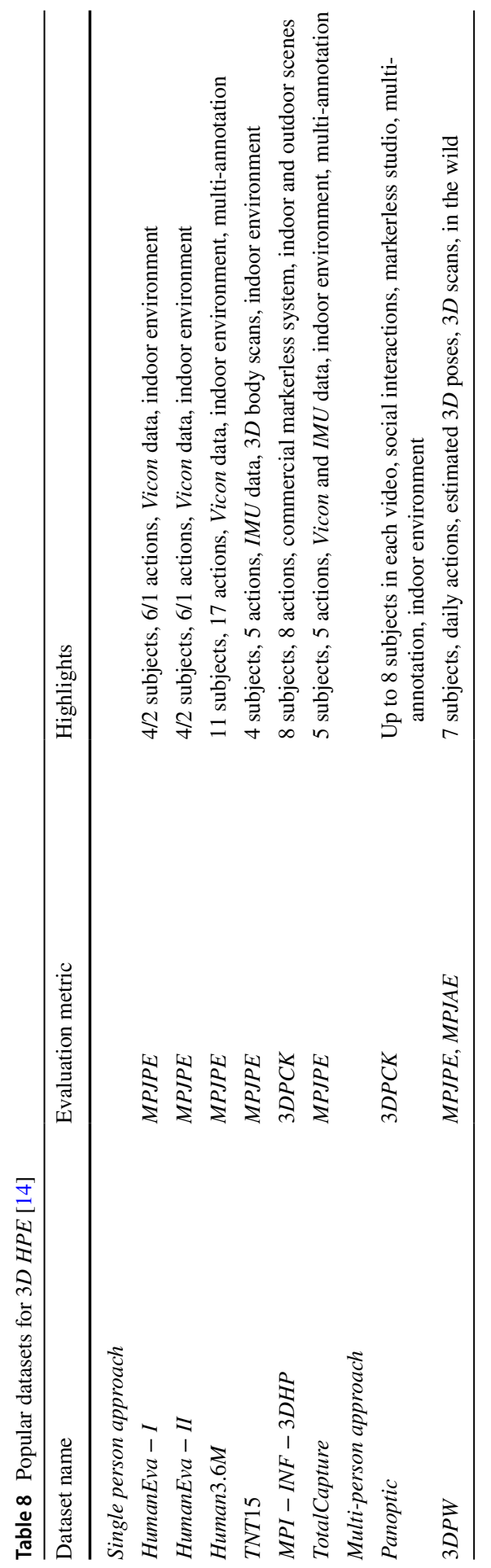


Percentage of Correct Key points $(P C K)$ measures the accuracy of the localization of the human body joints. A human body joint is considered correct if it falls within the threshold pixels of the ground-truth joint. Moreover, with the variation in a threshold, Area Under the Curve $(A U C)$ can be generated for further analysis.

The Object Key point Similarity $(O K S)$ and Average Precision $(A P)$ of $O K S$ consider scale and introduce the per-point constant to control falloff.

$A P$, Average Recall $(A R)$ and their variants are also metrics used in evaluating multi-person pose estimation results. $A P, A R$ and their variants are reported based on an analogous similarity measure: object key point similarity $(O K S)$, which plays the same role as the Intersection over Union ( $I o U)$. In addition, $A P / A R$ with different human body scales are also reported in the $C O C O$ dataset.

\subsection{Evaluation metrics used in 3D HPE}

There are several evaluation metrics for $3 D H P E$ with different limitation factors. In this subsection, we will give a list of widely used evaluation metrics.

Mean Per Joint Position Error (MPJPE) is one of the most popular metrics to evaluate the performance of $3 D H P E$. It is based on Euclidean distance and calculates the distance from the estimated $3 D$ joints to the ground truth, averaged over all joints in one image. In the set of frames cases, the mean error is averaged over all frames. Different datasets and protocols have different data post-processing of estimated joints before computing the MPJPE.

PMPJPE measure called a Reconstruction Error is the MPJPE after rigid alignment by post-processing between the estimated pose and the ground-truth one.

NMPJPE is defined as the MPJPE after normalizing the predicted positions in scale to the reference.

Mean Per Vertex Error (MPVE) measures the Euclidean distances between the ground truth vertices and the predicted vertices.

$3 D P C K$ is a $3 D$ extended version of the $P C K$ metric used in $2 D H P E$ evaluation. An estimated joint is considered correct if the distance between the estimation and the ground truth is within a certain threshold, and mainly the threshold is set to 150 $\mathrm{mm}$.

\section{Open issues and challenges}

$H P E$ is still a hot topic in computer vision which recently has evolved along with DL approaches. Despite the significant development of $2 D$ and $3 D$ human hand, pose and mesh estimation with $D L$, some unresolved open issues and challenges still exist between academia and industry, for example issue of the influence of human body part occlusion and crowded people. Effective practical models and sufficient training data are essential for $D L$-based methods. The massive interest in $H P E$ and its importance can be seen from the workshops and challenges on $H P E$, increasing. They gather researchers from academia and industry on HPE and discuss the current state-of-the-art and future research directions. Here we give some of them 
as we decide as recent and important ones: ICCV 2017-PoseTrack Challenge: Human Pose Estimation and Tracking in the Wild, CVPR 2018-3D humans 2018: 1st International workshop on Human pose, motion, activities and shape, ECCV 2018-PoseTrack Challenge: Articulated People Tracking in the Wild, CVPR 2019Workshop On Augmented Human: Human-centric Understanding, CVPR 2019-3D humans 2019: 2nd International workshop on Human pose, motion, activities and shape, ACM Multimedia-2020 Large-scale Human-centric Video Analysis in Complex Events, CVPR 2020-Towards Human-Centric Image/Video Synthesis, ECCV 2020-3D poses in the wild challenge.

\section{Conclusion}

This paper reviewed and discussed recent published $D L$-based papers on the human pose, hand and mesh estimation approaches in great detail. We have comprehensively investigated the related theoretical and practical issues compared to existing methods in this HPE research field. Moreover, the pose estimation concepts and their applications are clearly explained in detail to provide readers with a deeper understanding of these topics. We also provided a clear taxonomy of the presented survey-based $2 D$ and $3 D$ pose, hand and mesh estimation, including single-person or multi-person, single-stage or double-stage categories. In addition, datasets and metrics used in the HPE research approaches are provided for both $2 D$ and $3 D$ HPE approaches. The taxonomy of the presented paper is based on the methodology, which includes single-person or multi-person, single-stage or double-stage pipelines. The comparisons are made among different frameworks and different pipelines of the HPE approaches. Moreover, we also summarized the datasets and evaluation metrics for $D L$-based $2 D$ and $3 D H P E$ approaches. We hope that the presented review work can motivate new research efforts to improve the $H P E$ approaches with large-scale applications such as non-verbal and remote communication, including hand and body motion, $V R, A R$, human action recognition and computer games.

\section{Future research directions}

Despite the remarkable success in the $H P E$ field, there are still various promising future directions to promote advances in $H P E$ research. Further, we point out some of them:

$3 D$ HPE is usually used in visual tracking and analysis fields. Existing $3 D$ human hand, pose and mesh estimation from the given videos is not smooth and continuous. It is because the evaluation metrics cannot evaluate the smoothness. Suitable framelevel evaluation metrics focusing on temporary consistency and action smoothness should be generated.

The slight noise can significantly affect the performance of the HPE network. $D L$-based networks in computer vision tasks are weak to adversarial attacks. The 
researches against adversarial attacks can improve the robustness of models and promote real-world HPE applications.

Acknowledgements This work was supported by the Ministry of Education of the Republic of Korea and the National Research Foundation of Korea NRF-2019S1A5C2A03081234 and Inha University.

Open Access This article is licensed under a Creative Commons Attribution 4.0 International License, which permits use, sharing, adaptation, distribution and reproduction in any medium or format, as long as you give appropriate credit to the original author(s) and the source, provide a link to the Creative Commons licence, and indicate if changes were made. The images or other third party material in this article are included in the article's Creative Commons licence, unless indicated otherwise in a credit line to the material. If material is not included in the article's Creative Commons licence and your intended use is not permitted by statutory regulation or exceeds the permitted use, you will need to obtain permission directly from the copyright holder. To view a copy of this licence, visit http://creativecommons.org/licen ses/by/4.0/.

\section{References}

1. Andriluka M, Iqbal U, Insafutdinov E, Pishchulin L, Milan A, Gall J, Schiele B (2018) Posetrack: a benchmark for human pose estimation and tracking. In: Proceedings of the IEEE Conference on Computer Vision and Pattern Recognition, pp 5167-5176

2. Averbukh V, Averbukh N, Vasev P, Gvozdarev I, Levchuk G, Melkozerov L, Mikhaylov I (2019) Metaphors for software visualization systems based on virtual reality. In: International Conference on Augmented Reality, Virtual Reality and Computer Graphics, pp 60-70. Springer

3. Babu SC (2018) A 2019 guide to Human Pose Estimation with Deep Learning. Accessed 3 Feb 2018. https://nanonets.com/blog/human-pose-estimation-2d-guide/

4. Baek S, Kim KI, Kim TK (2019) Pushing the envelope for RGB-based dense 3d hand pose estimation via neural rendering. In: Proceedings of the IEEE Conference on Computer Vision and Pattern Recognition, pp 1067-1076

5. Barreda M, Dolz MF, Castaño MA, Alonso-Jordá P, Quintana-Orti ES (2020) Performance modeling of the sparse matrix-vector product via convolutional neural networks. J Supercomput 76(11):8883-8900

6. Bogo F, Kanazawa A, Lassner C, Gehler P, Romero J, Black MJ (2016) Keep it SMPL: automatic estimation of $3 \mathrm{~d}$ human pose and shape from a single image. In: European Conference on Computer Vision, pp 561-578. Springer

7. Boukhayma A, Bem R, Torr PH (2019) $3 \mathrm{~d}$ hand shape and pose from images in the wild. In: Proceedings of the IEEE Conference on Computer Vision and Pattern Recognition, pp 10843-10852

8. Bounouni M, Bouallouche-Medjkoune L (2018) Acknowledgment-based punishment and stimulation scheme for mobile ad hoc network. J Supercomput 74(10):5373-5398

9. Bulat A, Tzimiropoulos G (2016) Human pose estimation via convolutional part heatmap regression. In: 14th European Conference on Computer Vision (EECV 2016), 8-16 October 2016. Amsterdam, Netherlands

10. Bulat A, Tzimiropoulos G (2016) Human pose estimation via convolutional part heatmap regression. In: European Conference on Computer Vision. Springer, pp 717-732

11. Bulat A, Tzimiropoulos G (2016) Human pose estimation via convolutional part heatmap regression. Lecture Notes in Computer Science pp 717-732. https://doi.org/10.1007/978-3-319-46478-7_ 44

12. Cao Z, Simon T, Wei SE, Sheikh Y (2017) Realtime multi-person 2d pose estimation using part affinity fields. In: Proceedings of the IEEE Conference on Computer Vision and Pattern Recognition, pp 7291-7299

13. Carreira J, Agrawal P, Fragkiadaki K, Malik J (2016) Human pose estimation with iterative error feedback. In: Proceedings of the IEEE Conference on Computer Vision and Pattern Recognition, pp 4733-4742 
14. Chen Y, Tian Y, He M (2020) Monocular human pose estimation: a survey of deep learning-based methods. Comput Vis Image Underst 192:102897. https://doi.org/10.1016/j.cviu.2019.102897

15. Chen Y, Wang Z, Peng Y, Zhang Z, Yu G, Sun J (2018) Cascaded pyramid network for multiperson pose estimation. In: Proceedings of the IEEE Conference on Computer Vision and Pattern Recognition, pp 7103-7112

16. Choi H, Moon G, Lee KM (2020) Pose2mesh: Graph convolutional network for 3d human pose and mesh recovery from a 2d human pose. arXiv preprint arXiv:2008.09047

17. Choi S, Kim C, Kang YS, Youm S (2021) Human behavioral pattern analysis-based anomaly detection system in residential space. J Supercomput 77:1-18

18. Chu X, Yang W, Ouyang W, Ma C, Yuille AL, Wang X (2017) Multi-context attention for human pose estimation. In: Proceedings of the IEEE Conference on Computer Vision and Pattern Recognition, pp 1831-1840

19. Clark A, Absher J (2018) Cyber-surveillance analysis for supercomputing environments. In: Surveillance in action. Springer, pp. 395-412

20. Dang Q, Yin J, Wang B, Zheng W (2019) Deep learning based 2d human pose estimation: a survey. Tsinghua Sci Technol 24(6):663-676. https://doi.org/10.26599/TST.2018.9010100

21. Deng X, Yang S, Zhang Y, Tan P, Chang L, Wang H (2017) Hand3d: hand pose estimation using 3d neural network. arXiv preprint arXiv:1704.02224

22. Ding P, Zhang J, Zhou H, Zou X, Wang M (2020) Pyramid context learning for object detection. J Supercomput 76:1-14

23. Du X, Kuang D, Ye Y, Li X, Chen M, Du Y, Wu W (2018) Comparative study of distributed deep learning tools on supercomputers. In: International Conference on Algorithms and Architectures for Parallel Processing. Springer, pp 122-137

24. Fang B, Fang L (2020) Concise feature pyramid region proposal network for multi-scale object detection. J Supercomput 76(5):3327-3337

25. Fieraru M, Khoreva A, Pishchulin L, Schiele B (2018) Learning to refine human pose estimation. In: 2018 IEEE/CVF Conference on Computer Vision and Pattern Recognition Workshops (CVPRW). https://doi.org/10.1109/cvprw.2018.00058

26. Ge L, Liang H, Yuan J, Thalmann D (2017) 3d convolutional neural networks for efficient and robust hand pose estimation from single depth images. In: Proceedings of the IEEE Conference on Computer Vision and Pattern Recognition, pp 1991-2000

27. Ge L, Liang H, Yuan J, Thalmann D (2018) Robust 3d hand pose estimation from single depth images using multi-view CNNs. IEEE Trans Image Process 27(9):4422-4436

28. Ge L, Ren Z, Li Y, Xue Z, Wang Y, Cai J, Yuan J (2019) 3d hand shape and pose estimation from a single RGB image. In: Proceedings of the IEEE/CVF Conference on Computer Vision and Pattern Recognition, pp 10833-10842

29. Habermann M, Xu W, Zollhofer M, Pons-Moll G, Theobalt C (2020) Deepcap: monocular human performance capture using weak supervision. In: Proceedings of the IEEE/CVF Conference on Computer Vision and Pattern Recognition, pp 5052-5063

30. Haque A, Peng B, Luo Z, Alahi A, Yeung S, Fei-Fei L (2016) Towards viewpoint invariant 3d human pose estimation. In: European Conference on Computer Vision. Springer, pp. 160-177

31. He K, Zhang X, Ren S, Sun J (2015) Deep residual learning for image recognition. CoRR arXiv: abs/1512.03385

32. Hesse N, Pujades S, Black M, Arens M, Hofmann U, Schroeder S (2019) Learning and tracking the $3 \mathrm{~d}$ body shape of freely moving infants from RGB-D sequences. IEEE Trans Pattern Analysis Mach Intell 42:2540

33. Hidalgo G, Raaj Y, Idrees H, Xiang D, Joo H, Simon T, Sheikh Y (2019) Single-network wholebody pose estimation. In: Proceedings of the IEEE International Conference on Computer Vision, pp 6982-6991

34. Huang S, Gong M, Tao D (2017) A coarse-fine network for keypoint localization. In: Proceedings of the IEEE International Conference on Computer Vision, pp 3028-3037

35. Ichimura T, Fujita K, Yamaguchi T, Hori M, Wijerathne L, Ueda N (2020) Fast multi-step optimization with deep learning for data-centric supercomputing. In: Proceedings of the 20204 th International Conference on High Performance Compilation, Computing and Communications, pp 7-13

36. Insafutdinov E, Pishchulin L, Andres B, Andriluka M, Schiele B (2016) Deepercut: a deeper, stronger, and faster multi-person pose estimation model. In: European Conference on Computer Vision. Springer, pp 34-50 
37. Jifara W, Jiang F, Rho S, Cheng M, Liu S (2019) Medical image denoising using convolutional neural network: a residual learning approach. J Supercomput 75(2):704-718

38. Kanazawa A, Black MJ, Jacobs DW, Malik J (2018) End-to-end recovery of human shape and pose. In: Proceedings of the IEEE Conference on Computer Vision and Pattern Recognition, pp 7122-7131

39. Kaster J (2020) Training convolutional neural network classifiers using simultaneous scaled supercomputing. Tech. rep., University of Dayton Dayton United States

40. Kato H, Ushiku Y, Harada T (2018) Neural 3d mesh renderer. In: Proceedings of the IEEE Conference on Computer Vision and Pattern Recognition, pp 3907-3916

41. Kaur M, Kaur G, Sharma PK, Jolfaei A, D Singh (2020) Binary cuckoo search metaheuristicbased supercomputing framework for human behavior analysis in smart home. J Supercomput 76(4):2479-2502

42. Kawana Y, Ukita N, Huang JB, Yang MH (2018) Ensemble convolutional neural networks for pose estimation. Comput Vis Image Underst 169:62-74. https://doi.org/10.1016/j.cviu.2017.12.005

43. Ke L, Chang MC, Qi H, Lyu S (2018) Multi-scale structure-aware network for human pose estimation. In: Proceedings of the European Conference on Computer Vision (ECCV), pp 713-728

44. Ke S, Xiao B, Liu D, Wang J (2019) Deep high-resolution representation learning for human pose estimation. pp 5686-5696. https://doi.org/10.1109/CVPR.2019.00584

45. Khamparia A, Gupta D, de Albuquerque VHC, Sangaiah AK, Jhaveri RH (2020) Internet of health things-driven deep learning system for detection and classification of cervical cells using transfer learning. J Supercomput 76(11):8590-8608. https://doi.org/10.1007/s11227-020-03159-4

46. Kim M, Pons-Moll G, Pujades S, Bang S, Kim J, Black MJ, Lee SH (2017) Data-driven physics for human soft tissue animation. ACM Trans Gr 36(4):1-12

47. Kim S, Jang SW, ho Park J, Kim G (2019) Robust hand pose estimation using visual sensor in IoT environment. J Supercomput 76:5382-5401

48. Kocabas M, Karagoz S, Akbas E (2018) Multiposenet: fast multi-person pose estimation using pose residual network. In: Proceedings of the European Conference on Computer Vision (ECCV), pp 417-433

49. Kolotouros N, Pavlakos G, Black MJ, Daniilidis K (2019) Learning to reconstruct 3d human pose and shape via model-fitting in the loop. In: Proceedings of the IEEE International Conference on Computer Vision, pp 2252-2261

50. Kolotouros N, Pavlakos G, Daniilidis K (2019) Convolutional mesh regression for single-image human shape reconstruction** supplementary material

51. Kolotouros N, Pavlakos G, Daniilidis K (2019) Convolutional mesh regression for single-image human shape reconstruction. In: Proceedings of the IEEE Conference on Computer Vision and Pattern Recognition, pp 4501-4510

52. Kondratyuk N, Smirnov G, Agarkov A, Osokin A, Nikolskiy V, Semenov A, Stegailov V (2019) Performance and scalability of materials science and machine learning codes on the state-of-art hybrid supercomputer architecture. In: Russian Supercomputing Days. Springer, pp 597-609

53. Lee D, Kang S, Choi K (2018) Compend: computation pruning through early negative detection for ReLU in a deep neural network accelerator. In: Proceedings of the 2018 International Conference on Supercomputing, pp. 139-148

54. Li J, Liu M, Ma D, Huang J, Ke M, Zhang T (2020) Learning shared subspace regularization with linear discriminant analysis for multi-label action recognition. J Supercomput 76(3):2139-2157

55. Loghman M, Kim J, Choi K (2018) Fast depth estimation using semi-global matching and adaptive stripe-based optimization. J Supercomput 74(8):3666-3684

56. Loper M, Mahmood N, Romero J, Pons-Moll G, Black MJ (2015) SMPL: a skinned multi-person linear model. ACM Trans Gr 34(6):1-16

57. von Marcard T, Henschel R, Black MJ, Rosenhahn B, Pons-Moll G (2018) Recovering accurate 3d human pose in the wild using imus and a moving camera. In: Proceedings of the European Conference on Computer Vision (ECCV), pp 601-617

58. Martinez J, Hossain R, Romero J, Little JJ (2017) A simple yet effective baseline for 3d human pose estimation. In: 2017 IEEE International Conference on Computer Vision (ICCV). https://doi. org/10.1109/iccv.2017.288

59. McDanel B, Zhang SQ, Kung H, Dong X (2019) Full-stack optimization for accelerating CNNs using powers-of-two weights with FPGA validation. In: Proceedings of the ACM International Conference on Supercomputing, pp 449-460 
60. Mehta D, Rhodin H, Casas D, Fua P, Sotnychenko O, Xu W, Theobalt C (2017) Monocular 3d human pose estimation in the wild using improved CNN supervision. In: 2017 International Conference on 3D Vision (3DV), IEEE, pp 506-516

61. Mehta D, Sotnychenko O, Mueller F, Xu W, Sridhar S, Pons-Moll G, Theobalt C (2017) Singleshot multi-person 3d pose estimation from monocular RGB. arXiv preprint arXiv:1712.03453

62. Mehta D, Sotnychenko O, Mueller F, Xu W, Sridhar S, Pons-Moll G, Theobalt C (2018) Singleshot multi-person 3d pose estimation from monocular RGB. In: 20182017 International Conference on 3D Vision (3DV), IEEE, pp 120-130

63. Millar K, Cheng A, Chew HG, Lim CC (2019) Using convolutional neural networks for classifying malicious network traffic. Springer, Cham, pp 103-126. https://doi.org/10.1007/ 978-3-030-13057-2_5

64. Moon G, Chang JY, Lee KM (2019) Camera distance-aware top-down approach for 3d multi-person pose estimation from a single RGB image. In: 2019 IEEE/CVF International Conference on Computer Vision (ICCV). https://doi.org/10.1109/iccv.2019.01023

65. Moon G, Chang JY, Lee KM (2019) Posefix: model-agnostic general human pose refinement network. In: Proceedings of the IEEE Conference on Computer Vision and Pattern Recognition, pp 7773-7781

66. Moon G, Lee KM (2020) I21-meshnet: Image-to-lixel prediction network for accurate 3d human pose and mesh estimation from a single RGB image. arXiv preprint arXiv:2008.03713

67. Moon G, Yong Chang J, Mu Lee K (2018) V2v-posenet: voxel-to-voxel prediction network for accurate $3 \mathrm{~d}$ hand and human pose estimation from a single depth map. In: Proceedings of the IEEE Conference on Computer Vision and Pattern Recognition, pp. 5079-5088

68. Mukhiddin T, Lee W, Lee S, Rashid T (2020) Research issues on generative adversarial networks and applications. In: 2020 IEEE International Conference on Big Data and Smart Computing (BigComp), IEEE, pp 487-488

69. Newell A, Huang Z, Deng J (2017) Associative embedding: end-to-end learning for joint detection and grouping. In: Advances in Neural Information Processing Systems, pp 2277-2287

70. Newell A, Yang K, Deng J (2016) Stacked hourglass networks for human pose estimation. In: European Conference on Computer Vision. Springer, pp 483-499

71. Nie X, Feng J, Zhang J, Yan S (2019) Single-stage multi-person pose machines. In: Proceedings of the IEEE/CVF International Conference on Computer Vision, pp 6951-6960

72. Oberweger M, Lepetit V (2017) Deepprior++: improving fast and accurate $3 d$ hand pose estimation. In: Proceedings of the IEEE International Conference on Computer Vision Workshops, pp 585-594

73. Oberweger M, Wohlhart P, Lepetit V (2015) Training a feedback loop for hand pose estimation. In: Proceedings of the IEEE International Conference on Computer Vision, pp 3316-3324

74. Oh SH, Han SW, Choi BS, Kim GW, Lim KS (2018) Deep feature learning for person re-identification in a large-scale crowdsourced environment. J Supercomput 74(12):6753-6765

75. de Oliveira CHR, Costa APF, Thomaz VF, Silva IA (2019) Low-cost deployment proposal to urban mobility in smart cities. J Supercomput 75(11):7265-7289

76. Oliveira D, Blanchard S, DeBardeleben N, Fernandes dos Santos F, Piscoya Dávila G, Navaux P, Favalli A, Schappert O, Wender S, Cazzaniga C et al (2020) Thermal neutrons: a possible threat for supercomputer reliability. J Supercomput 77:1-23

77. Omran M, Lassner C, Pons-Moll G, Gehler P, Schiele B (2018) Neural body fitting: unifying deep learning and model based human pose and shape estimation. In: 2018 International Conference on 3D Vision (3DV), IEEE, pp. 484-494

78. Pan H, Li Y, Zhao D (2021) Recognizing human behaviors from surveillance videos using the SSD algorithm. J Supercomput. https://doi.org/10.1007/s11227-020-03578-3

79. Panteleris P, Oikonomidis I, Argyros A (2018) Using a single RGB frame for real time $3 \mathrm{~d}$ hand pose estimation in the wild. In: 2018 IEEE Winter Conference on Applications of Computer Vision (WACV), IEEE, pp 436-445

80. Papandreou G, Zhu T, Kanazawa N, Toshev A, Tompson J, Bregler C, Murphy K (2017) Towards accurate multi-person pose estimation in the wild. In: Proceedings of the IEEE Conference on Computer Vision and Pattern Recognition, pp 4903-4911

81. Papandreou G, Zhu T, Kanazawa N, Toshev A, Tompson J, Bregler C, Murphy K (2017) Towards accurate multi-person pose estimation in the wild. In: 2017 IEEE Conference on Computer Vision and Pattern Recognition (CVPR). https://doi.org/10.1109/cvpr.2017.395 
82. Park S, Hwang J, Kwak N (2016) 3d human pose estimation using convolutional neural networks with 2d pose information. In: Computer Vision-ECCV 2016 Workshops, pp. 156-169. https:// doi.org/10.1007/978-3-319-49409-8_15

83. Pavlakos G, Zhou X, Derpanis KG, Daniilidis K (2017) Coarse-to-fine volumetric prediction for single-image 3d human pose. In: 2017 IEEE Conference on Computer Vision and Pattern Recognition (CVPR). https://doi.org/10.1109/cvpr.2017.139

84. Pishchulin L, Insafutdinov E, Tang S, Andres B, Andriluka M, Gehler P, Schiele B (2016) Deepcut: joint subset partition and labeling for multi person pose estimation. In: Proceedings of the IEEE Conference on Computer Vision and Pattern Recognition, pp 4929-4937

85. Proietti R, Shang Y, Xiao X, Chen X, Zhang Y, Yoo SB (2019) Self-driving reconfigurable silicon photonic interconnects (flex-lions) with deep reinforcement learning. Supercomput Poster, 118

86. Prokudin S, Black MJ, Romero J (2020) Smplpix: neural avatars from 3d human models. arXiv preprint arXiv:2008.06872

87. Pujades S, Mohler B, Thaler A, Tesch J, Mahmood N, Hesse N, Bülthoff HH, Black MJ (2019) The virtual caliper: rapid creation of metrically accurate avatars from $3 \mathrm{~d}$ measurements. IEEE Trans Vis Comput Gr 25(5):1887-1897

88. Ramírez I, Cuesta-Infante A, Schiavi E, Pantrigo JJ (2020) Bayesian capsule networks for 3d human pose estimation from single $2 \mathrm{~d}$ images. Neurocomputing 379:64-73. https://doi.org/10. 1016/j.neucom.2019.09.101

89. Rane C, Mehrotra R, Bhattacharyya S, Sharma M, Bhattacharya M (2020) A novel attention fusion network-based framework to ensemble the predictions of CNNs for lymph node metastasis detection. J Supercomput 77:1-20

90. Rhodin H, Salzmann M, Fua P (2018) Unsupervised geometry-aware representation for 3d human pose estimation. In: Proceedings of the European Conference on Computer Vision (ECCV), pp 750-767

91. Rogez G, Weinzaepfel P, Schmid C (2017) LCR-Net: localization-classification-regression for human pose. In: Proceedings of the IEEE Conference on Computer Vision and Pattern Recognition, pp 3433-3441

92. Romero J, Tzionas D, Black MJ (2017) Embodied hands: modeling and capturing hands and bodies together. ACM Trans Gr 36(6):245

93. Sabour S, Frosst N, Hinton G (2017) Dynamic routing between capsules

94. Sattar H, Krombholz K, Pons-Moll G, Fritz M (2019) Shape evasion: preventing body shape inference of multi-stage approaches. arXiv preprint arXiv:1905.11503

95. Sharma S, Varigonda PT, Bindal P, Sharma A, Jain A (2019) Monocular 3d human pose estimation by generation and ordinal ranking. In: Proceedings of the IEEE International Conference on Computer Vision, pp 2325-2334

96. Sharma V, Srinivasan K, Kumar R, Chao HC, Hua KL (2017) Efficient cooperative relaying in flying ad hoc networks using fuzzy-bee colony optimization. J Supercomput 73(7):3229-3259

97. Shi D, Wei X, Yu X, Tan W, Ren Y, Pu S (2021) Inspose: instance-aware networks for single-stage multi-person pose estimation. arXiv preprint arXiv:2107.08982

98. Singha T, Pham DS, Krishna A, Dunstan J (2020) Efficient segmentation pyramid network. In: International Conference on Neural Information Processing. Springer, pp 386-393

99. Sun K, Xiao B, Liu D, Wang J (2019) Deep high-resolution representation learning for human pose estimation. In: Proceedings of the IEEE Conference on Computer Vision and Pattern Recognition, pp 5693-5703

100. Sun X, Shang J, Liang S, Wei Y (2017) Compositional human pose regression. In: 2017 IEEE International Conference on Computer Vision (ICCV). https://doi.org/10.1109/iccv.2017.284

101. Sun X, Wei Y, Liang S, Tang X, Sun J (2015) Cascaded hand pose regression. In: Proceedings of the IEEE Conference on Computer Vision and Pattern Recognition, pp 824-832

102. Sun X, Xiao B, Wei F, Liang S, Wei Y (2018) Integral human pose regression. In: Proceedings of the European Conference on Computer Vision (ECCV), pp 529-545

103. Tang D, Chang HJ, Tejani A, Kim TK (2016) Latent regression forest: structured estimation of 3d hand poses. IEEE Trans Pattern Anal Mach Intell 39(7):1374-1387

104. Tekin B, Katircioglu I, Salzmann M, Lepetit V, Fua P (2016) Structured prediction of 3d human pose with deep neural networks. In: Proceedings of the British Machine Vision Conference 2016. https://doi.org/10.5244/c.30.130

105. Toshev A, Szegedy C (2014) Deeppose: human pose estimation via deep neural networks. In: Proceedings of the IEEE Conference on Computer Vision and Pattern Recognition, pp 1653-1660 
106. Toshpulatov M, Lee W, Lee S (2021) Generative adversarial networks and their application to $3 \mathrm{~d}$ face generation: a survey. Image Vis Comput 108:104119. https://doi.org/10.1016/j.imavis.2021. 104119

107. Toutouh J, Esteban M, Nesmachnow S (2020) Parallel/distributed generative adversarial neural networks for data augmentation of covid-19 training images. In: Latin American High Performance Computing Conference. Springer, pp 162-177

108. Tseng KK, Zhang R, Chen CM, Hassan MM (2020) Dnetunet: a semi-supervised CNN of medical image segmentation for super-computing AI service. J Supercomput 77:1-22

109. Verma N, Boyer E, Verbeek J (2018) Feastnet: feature-steered graph convolutions for 3d shape analysis. In: Proceedings of the IEEE Conference on Computer Vision and Pattern Recognition, pp 2598-2606

110. Wan C, Probst T, Van Gool L, Yao A (2017) Crossing nets: Combining GANs and VAEs with a shared latent space for hand pose estimation. In: Proceedings of the IEEE Conference on Computer Vision and Pattern Recognition, pp 680-689

111. Wang N, Zhang Y, Li Z, Fu Y, Liu W, Jiang YG (2018) Pixel2mesh: generating 3d mesh models from single RGB images. In: Proceedings of the European Conference on Computer Vision (ECCV), pp 52-67

112. Wang Z, Peng Y, Yu ZZG, Sun J et al. (2018) Cascaded pyramid network for multi-person pose estimation

113. Wu E, Koike H (2019) Futurepose-mixed reality martial arts training using real-time 3d human pose forecasting with a RGB camera. In: 2019 IEEE Winter Conference on Applications of Computer Vision (WACV), IEEE, pp 1384-1392

114. Xiang D, Joo H, Sheikh Y (2019) Monocular total capture: posing face, body, and hands in the wild. In: Proceedings of the IEEE Conference on Computer Vision and Pattern Recognition, pp 10965-10974

115. Xiao B, Wu H, Wei Y (2018) Simple baselines for human pose estimation and tracking. In: Proceedings of the European Conference on Computer Vision (ECCV), pp 466-481

116. Xiao SSJ (2016) Deep sliding shapes for amodal 3d object detection in RGB-D images. In: Proceedings of the IEEE Conference on Computer Vision and Pattern Recognition, pp 808-816

117. Xiao Y, Yu D, Wang X, Lv T, Fan Y, Wu L (2020) Spcnet: spatial preserve and content-aware network for human pose estimation. In: ECAI

118. Yang W, Ouyang W, Wang X, Ren J, Li H, Wang X (2018) 3d human pose estimation in the wild by adversarial learning. In: 2018 IEEE/CVF Conference on Computer Vision and Pattern Recognition. https://doi.org/10.1109/cvpr.2018.00551

119. Ye Q, Yuan S, Kim TK (2016) Spatial attention deep net with partial PSO for hierarchical hybrid hand pose estimation. In: European Conference on Computer Vision. Springer, pp 346-361

120. Yong B, Zhang G, Chen H, Zhou Q (2017) Intelligent monitor system based on cloud and convolutional neural networks. J Supercomput 73(7):3260-3276

121. Yuan S, Ye Q, Garcia-Hernando G, Kim TK (2017) The 2017 hands in the million challenge on $3 \mathrm{~d}$ hand pose estimation. arXiv preprint arXiv: 1707.02237

122. Yun K, Park J, Cho J (2020) Robust human pose estimation for rotation via self-supervised learning. IEEE Access 8:32502-32517. https://doi.org/10.1109/ACCESS.2020.2973390

123. Zhao L, Peng X, Tian Y, Kapadia M, Metaxas DN (2019) Semantic graph convolutional networks for $3 \mathrm{~d}$ human pose regression. In: Proceedings of the IEEE Conference on Computer Vision and Pattern Recognition, pp 3425-3435

124. Zheng C, Wu W, Yang T, Zhu S, Chen C, Liu R, Shen J, Kehtarnavaz N, Shah M (2020) Deep learning-based human pose estimation: a survey. ArXiv arXiv:abs/2012.13392

125. Zhou X, Huang Q, Sun X, Xue X, Wei Y (2017) Towards 3d human pose estimation in the wild: a weakly-supervised approach. In: 2017 IEEE International Conference on Computer Vision (ICCV). https://doi.org/10.1109/iccv.2017.51

126. Zhou X, Wan Q, Zhang W, Xue X, Wei Y (2016) Model-based deep hand pose estimation. arXiv: 1606.06854

127. Zimmermann C, Ceylan D, Yang J, Russell B, Argus M, Brox T (2019) Freihand: A dataset for markerless capture of hand pose and shape from single RGB images. In: Proceedings of the IEEE International Conference on Computer Vision, pp 813-822 
Publisher's Note Springer Nature remains neutral with regard to jurisdictional claims in published maps and institutional affiliations.

\section{Authors and Affiliations}

Mukhiddin Toshpulatov ${ }^{1}$ D $\cdot$ Wookey Lee ${ }^{1} \cdot$ Suan Lee $^{2} \cdot$ Arousha Haghighian Roudsari ${ }^{3}$

Mukhiddin Toshpulatov

muhiddin1979@inha.edu

Suan Lee

suanlee@semyung.ac.kr

Arousha Haghighian Roudsari

arousha.haghighian@inha.edu

1 Biomedical Science and Engineering, Inha University, 100 Inha-ro, Michuhol-gu, Incheon 22212, South Korea

2 School of Computer Science, Semyung University, Jecheon 27136, South Korea

3 Industrial Engineering, Inha University, 100 Inha-ro, Michuhol-gu, Incheon 22212, South Korea 